Die Schweiz und die humanitäre Aktion

\title{
1. Internationale Finanzbeziehungen
}

\section{Bruno Gurtner}

\section{OpenEdition}

\section{Journals}

Electronic version

URL: http://journals.openedition.org/sjep/646

DOI: $10.4000 /$ sjep.646

ISSN: 1663-9677

\section{Publisher}

Institut de hautes études internationales et du développement

\section{Printed version}

Date of publication: 1 janvier 1999

Number of pages: $99-125$

ISSN: $1660-5926$

\section{Electronic reference}

Bruno Gurtner, «1. Internationale Finanzbeziehungen », Schweizerisches Jahrbuch für

Entwicklungspolitik [Online], 18 | 1999, Online erschienen am: 28 August 2012, abgerufen am 08 September 2020. URL : http://journals.openedition.org/sjep/646 ; DOI : https://doi.org/10.4000/sjep. 646

(c) The Graduate Institute 


\section{INTERNATIONALE FINANZBEZIEHUNGEN*}

$H$ AUPTTHEMA des turbulenten verflossenen Jahres war die Finanzkrise. Ab Mitte 1997 in einigen asiatischen Tigerländern offen ausgebrochen, vertiefte sie sich und breitete sich über das ohnehin mit riesigen innenpolitischen Problemen belastete Russland und weitere GUS- und osteuropäische Staaten aus. Südafrika und einige lateinamerikanische Staaten blieben nicht verschont. Japans Wirtschafts- und Finanzkrise beeinträchtigte durchgreifende Massnahmen. Die nördlichen Industriestaaten befürchten ein Durchschlagen auf den Gang ihrer Wirtschaft. Grosse Finanzinstitute erlitten teils massive Verluste. Die Finanzkrise verursachte eine massive Umlagerung der Finanzströme. Sowohl die privaten wie die öffentlichen Finanzleistungen in Entwicklungs- und Transitionsländer sanken beträchtlich. Die multilateralen Finanzierungsinstitutionen mussten rekordmässige Überbrückungskredite bereitstellen. Dabei geriet insbesondere der Internationale Währungsfonds (IWF) mit seiner Strategie und seiner Rolle arg unter Druck.

\subsection{FINANZFLÜSSE IN DIE ENTWICKLUNGS- UND TRANSITIONSLÄNDER}

Nach mehreren Jahren insgesamt steigender Finanzflüsse in die Entwicklungsund Transitionsländer hat sich der Trend gekehrt. Gemäss der Organisation für wirtschaftliche Zusammenarbeit und Entwicklung (OECD) fielen die Finanzflüsse von 368 Milliarden Dollar im Jahre 1996 auf 272 Milliarden im Jahre $1997^{1}$ (vgl. Tabelle Nr. 1).

Sowohl das Volumen wie auch die Arten der Finanzflüsse variieren sehr stark von Region zu Region. So erhielt Afrika südlich der Sahara 1997 pro Kopf der Bevölkerung 27 Dollar an Entwicklungshilfe und bloss 3 Dollar an ausländische Direktinvestitionen. Demgegenüber flossen 13 Dollar Hilfe und 62 Dollar Direktinvestitionen in die Länder Lateinamerikas und der Karibik.

In ihrem Bericht Global Development Finance 1998 weist die Weltbank im Gegensatz zur OECD für 1997 gegenüber dem Vorjahr provisorisch noch eine leichte Zunahme der Netto-Ressourcenzuflüsse um 18,7 Milliarden auf 300,3 Milliarden Dollar aus². Aber die Weltbank erwartet für 1998 ebenfalls spärlicher fliessende Kapitalströme. Das erwarten auch IWF, UNCTAD, die Bank für Internationalen Zahlungsausgleich in Basel (BIZ) und das Washingtoner Institute of International Finance (IIF), ein Think Tank der grossen privaten internationalen Geschäftsbanken.

* Von Bruno Gurtner, Ökonom, Arbeitsgemeinschaft der Hilfswerke, Bern.

1. OECD, New Release, Aid and Private Flows Fell in 1997, Paris, 18. Juni 1998. Die ausgewiesenen Zahlen differieren zu jenen der Vorjahresstatistik, weil sowohl die Liste der Empfänger- wie der Geberstaaten Änderungen zu verzeichnen hatten.

2. The World Bank, Global Development Finance 1998, Washington 1998. Zu unterschiedlichen Datenerhebungen in OECD, Weltbank, IWF, UNCTAD und BIZ JSDW 1997, S. 13/14. 
Tabelle Nr. 1: Nettokapitalströme in die Entwicklungs- und Transitionsländer (in Mrd. \$ zu laufenden Preisen)

\begin{tabular}{lrrr}
\hline & $\mathbf{1 9 9 5}$ & $\mathbf{1 9 9 6}$ & $\mathbf{1 9 9 7}^{\mathbf{p}}$ \\
\hline I. Öffentliche Entwicklungsfinanzierung & $\mathbf{8 9 . 3}$ & $\mathbf{7 8 . 1}$ & $\mathbf{7 0 . 0}$ \\
\hline 1. Staatliche Entwicklungshilfe & 59.8 & 57.9 & 49.0 \\
\hline davon: Bilateral & 40.6 & 39.1 & 31.0 \\
\hline \multicolumn{1}{|c}{ Multilateral } & 19.2 & 18.9 & 18.0 \\
\hline 2. Andere Entwicklungsfinanzierung & 21.1 & 14.5 & 14.0 \\
\hline davon: Bilateral & 14.0 & 5.8 & 6.0 \\
\hline$\quad$ Multilateral & 7.1 & 8.7 & 8.0 \\
\hline II. Exportkredite & $\mathbf{5 . 6}$ & $\mathbf{4 . 0}$ & $\mathbf{- 4 . 4}$ \\
\hline III. Private Finanzströme & $\mathbf{1 6 8 . 3}$ & $\mathbf{2 8 6 . 3}$ & $\mathbf{2 0 6 . 2}$ \\
\hline 1. Direktinvestitionen & 52.3 & 64.3 & $\mathbf{7 5 . 0}$ \\
\hline 2. Internationale Bankkredite & 76.9 & 86.0 & 12.0 \\
\hline 3. Internationale Anleihen & 30.0 & 96.6 & 83.2 \\
\hline 4. Andere** & 3.1 & 33.8 & 30.0 \\
\hline 5. Private Hilfswerke & 6.0 & 5.6 & 6.0 \\
\hline Total Netto-Zuflüsse & $\mathbf{2 6 3 . 2}$ & $\mathbf{3 6 8 . 4}$ & $\mathbf{2 7 1 . 8}$ \\
\hline
\end{tabular}

Quelle: OECD Press Release, 18. Juni 1998

* Ohne Obligationen-Anleihen der Banken (in Rubrik III.3 enthalten) und ohne garantierte Kredite (in Rubrik II enthalten).

** Verschiedene DAC-Länder berichten nur unvollständig. Ab 1996 ist Japan eingeschlossen.

${ }^{\mathrm{P}}$ provisorische Angaben

\section{$\square$ Rückläufige öffentliche Entwicklungsfinanzierung}

Gemäss OECD erreichte die öffentliche Entwicklungshilfe ihrer Mitgliedsländer 1997 bloss noch 0,22\% ihres Bruttosozialproduktes (BSP), der tiefste je gemessene Wert. Negativ zu Buche schlagen mit Ausnahme Kanadas insbesondere die grossen Länder der Gruppe der Sieben (G-7), deren BSP-Anteil jetzt auf 0,19\% gesunken ist. Der entsprechende Wert der übrigen OECD-Länder beträgt 0,45\%. In realen Werten - korrigiert um Inflation und Wechselkursveränderungen - stieg die öffentliche Entwicklungszusammenarbeit in 11 von 14 Ländern, die der G-7 nicht angehören. Die Schweiz weist noch 0,32\% auf gegenüber 0,34 im Vorjahr. Das entspricht einem inflations- und wechselkursbereinigten Rückgang um 4,2\%.

凹SDW 1999, Kapitel 8, Abschnitt 8.1.

Die Weltbank ${ }^{3}$ weist angesichts der knapperen Entwicklungsbudgets der Geberländer auf die weiterhin verstärkten Bemühungen um Effektivitäts- und Effizienzsteigerung der Entwicklungszusammenarbeit hin. Mehr und mehr werden die Mittel in Länder mit einer reformfreudigen Politik gelenkt. Offizielle Agenturen würden auch innovative Massnahmen treffen, um den Zufluss langfristiger Privatkapitalflüsse zu fördern. Diesen Themen ist auch der OECD-Jahresbericht 1997 gewidmet $^{4}$. Der IWF seinerseits verweist ${ }^{5}$ auf die Tatsache hin, dass die ärmeren Entwicklungsländer nach wie vor auf konzessionelle, öffentliche Leistungen angewiesen bleiben.

Der Rückgang nichtkonzessioneller Mittel in den letzten Jahren ist mit der Bereitstellung umfangreicher Überbrückungskredite an Krisenländer gegen Ende 1997 umgekehrt worden.

3. The World Bank, Globald Development Finance 1998, aaO. S. 5 und $49 \mathrm{ff}$.

4. OECD, Entwicklungszusammenarbeit. Politik und Leistungen der Mitglieder des Ausschusses für Entwicklungshilfe. Bericht 1997, Paris 1998.

5. IMF, Official Financing for Developing Countries, Washington, Februar 1998. 


\section{$\square$ Private Kapitalflüsse: Wachstumstrend gebrochen}

Seit 1990 sind die privaten Kapitalflüsse in die Entwicklungs- und Transitionsländer ständig stärker gewachsen. Mit dem Ausbruch der Finanzkrise in Südostasien Mitte 1997 ist dieser Trend gebrochen. Die OECD weist für das ganze Jahr 1997 mit 206 Milliarden Dollar bereits einen um 80 Milliarden Dollar kleineren Zustrom aus als im Vorjahr (siehe Tabelle Nr. 1). Gemäss $\mathrm{OECD}^{6}$ vermochte der erneut gestiegene Zustrom der Privatkapitalflüsse nach Lateinamerika die rückläufigen Bankenkredite für Asien nicht zu kompensieren. Direkte Auslandsinvestitionen stiegen gesamthaft weiterhin an, während alle übrigen Formen privater Kapitalflüsse teils markant kleiner waren als im Vorjahr. Laut diesen provisorischen OECD-Zahlen fliesst der Hauptteil nach wie vor in die wirtschaftlich dynamischeren Länder Asiens, Europas und Südamerikas. In die Gruppe der ärmeren Entwicklungsländer flossen total 22 Milliarden Dollar Privatkapital, jedoch konzentriert auf Indien und China. Die Länder Afrikas südlich der Sahara erhielten zusammen bloss je 2 Milliarden Dollar an Direktinvestitionen und Bankenkredite.

Diesen Trendwechsel bestätigen auch andere Statistiken. Gemäss Schätzungen des Washingtoner Institute of International Finance $(I I F)^{7}$ überstiegen zwar die Direktinvestitionen 1997 nochmals die Vorjahreszahlen. Hingegen blieben die Portfolioanlagen deutlich hinter den 1996 erreichten Werten zurück, und die Bankenkredite waren gar negativ, d.h. es floss mehr zurück als neu ausgeliehen wurde.

Das bestätigt auch die Bank für Internationalen Zahlungsausgleich (BIZ). «Die Nettozuflüsse internationalen Kapitals, grösstenteils in Form kurzfristiger Bankkredite, stiegen von praktisch null im Jahr 1989 auf einen Höchstwert von fast \$170 Milliarden im Jahr 1996, bis sie in jüngster Zeit durch beträchtliche Nettokapitalabflüsse abgelöst wurden ${ }^{8}$.» Die BIZ-Quartalsstatistiken verdeutlichen den dramatischen Umschwung für die fünf am meisten betroffenen asiatischen Länder: Interbanken-Ausleihen und Ausleihen an Nichtbanken stiegen zwischen dem vierten Quartal 1996 und dem dritten Quartal 1997 noch um 22 Milliarden Dollar. Im letzten Quartal 1997 flossen jedoch 32 Milliarden und im ersten Quartal 1998 nochmals 34 Milliarden Dollar zurück.

Die Weltbank ${ }^{9}$ weist hingegen für 1997 mit 256 Milliarden Dollar nochmals einen, verglichen mit den 247 Milliarden des Vorjahres, grösseren privaten Kapitalfluss aus. Das Wachstum ist allerdings deutlich langsamer als in den Vorjahren. Dazu haben alle Formen der privaten Kapitalflüsse beigetragen. (Siehe JSDW 1998, S. 140, Kasten «Private Kapitalflüsse»). Einzig die Portfolioinvestitionen übertrafen das Vorjahresniveau nicht mehr und erreichten bloss noch die gleiche Höhe wie in den Jahren 1994 und 1995. Die Weltbank publiziert früher als andere Institutionen ihre provisorischen neuesten Zahlen, die jedoch auf weiter zurückliegenden Statistiken beruhen. (Siehe unter Kasten «BIZ-Statistiken zum internationalen Bankgeschäft»). Aber die Weltbank verweist bereits deutlich darauf, dass in naher Zukunft die Privatkapitalströme abnehmen werden. Direktinvestitionen dürften dabei weniger betroffen sein als Portfolioinvestitionen, Bonds und Bankanleihen.

6. OECD, News Release, 18. Juni 1998, aaO.

7. Zitiert nach Neuer Zürcher Zeitung, «Abrupt versiegende Kapitalströme im Jahre 1997», 30. Januar 1998.

8. Bank für Internationalen Zahlungsausgleich (BIZ), 68. Jahresbericht, Basel, 1998, S. 187.

9. The World Bank, Global Development Finance 1998, aaO.,Tabelle 1. 
Trotz zunehmender Volatilität und Unsicherheit stellt die Weltbank allerdings eine vertiefte Teilnahme zahlreicher Entwicklungs- und Transitionsländer auf den internationalen Finanzmärkten mit teils innovativen Formen fest.

\section{$\square$ Die Finanzkrise}

Die derzeitige Finanzkrise hat zu einer unüberschaubaren Menge an Publikationen geführt. Internationale Finanzierungsinstitutionen, Regierungen, Geschäftsbanken, Medien, Wissenschaft, Nichtregierungsorganisationen usw. haben zahllose Seiten Papier gefüllt über Ursachen, Verlauf, künftige Entwicklung, zu treffende Massnahmen, Kritik an Institutionen usw. Die nachstehende Übersicht bleibt notwendigerweise beschränkt.

$\llbracket$ JSDW 1998, S. $141 \mathrm{ff}$. Die Finanzkrise in den Schwellenländern Asiens.

Die Finanzkrise Ostasiens, offen aufgetreten im Juli 1997 in Thailand, weitete sich unaufhaltsam auf andere Länder und Regionen aus. Betroffen waren neben den andern ostasiatischen Schwellenländern auch China und Japan, Russland, weitere GUS-Staaten und osteuropäische Länder. Zuletzt bedrohte die Krise auch südamerikanische Länder (Brasilien, Venezuela, Argentinien) sowie auch Südafrika. Zumindest teilweise förderten interne Probleme in einzelnen Ländern die Ausbreitung der Finanzkrise.

Es sei nicht völlig sicher, so die BIZ ${ }^{10}$, dass inzwischen das Schlimmste überstanden sei. Noch nicht in vollem Umfang abzusehen seien die wirtschaftlichen und sozialen Kosten. Es bleibe abzuwarten, wie stark inländische Unternehmen und ihre Kreditgeber in Mitleidenschaft gezogen worden seien. Der Schock war um so grösser, weil Asien als Zukunftsmodell gegolten hatte. Immer zahlreichere Kommentatoren machten hinter der Liquiditätskrise eine namhafte Strukturkrise aus.

Die BIZ nennt für Südostasien klar binnenwirtschaftliche Ursachen der Krise: ein übermässiges Kreditwachstum insbesondere des privaten Sektors mit kurzfristigen Verbindlichkeiten, damit verbunden eine übermässige Ausweitung des Kapitalstocks, unzureichend beaufsichtigte Banken- und Finanzmarktsysteme, Preisblasen bei Vermögenswerten und allzu starre Wechselkursbindungen, welche eine flexiblere Geldpolitik verhinderten. Die Dollaraufwertung ab 1995 und sinkende Exportpreise, insbesondere für Elektronikprodukte, haben diese Länder anfällig gemacht für kompetitive Abwertungen. Vermutete Schwächen im Finanzsektor sind infolge unzureichender Transparenz von den internationalen Märkten nicht rechtzeitig und in genügendem Ausmass wahrgenommen worden. Hingegen blieben Inflation und Staatsdefizite gering.

Es wäre aber ein Fehler, so die BIZ, daraus den Schluss zu ziehen, dass bei einer besseren Wirtschaftspolitik in den einzelnen Ländern alle Schwierigkeiten leicht vermeidbar gewesen wären. Internationale makroökonomische Ungleichgewichte hätten wesentlich zu den Entwicklungen in Asien beigetragen und würden sich möglicherweise auch noch auf andere Weise manifestieren.

Insbesondere nennt die BIZ die unerwartet hartnäckigen Konkjunkturunterschiede zwischen den grossen Industrieländern sowie die weltweite entschlos-

10. BIZ, 68. Jahresbericht, aaO. S. $3 \mathrm{ff}$. 
sene stabilitätsorientierte Geldpolitik mit tiefen Zinsen als Ursachen für eine starke Ausweitung der weltweiten Liquidität. Das hat vorerst zum bekannten Anschwellen der Finanzflüsse in die aufstrebenden Märkte geführt. Die Anleger seien, so die BIZ, in ihrem Streben nach Wahrung der Renditen versucht gewesen, die gestiegenen Risiken nicht ausreichend zu beachten.

Nach Ausbruch der Finanzkrise haben die Anleger in grossem Ausmass versucht, ihre Gelder aus den bedrohten Märkten zurückzuziehen (siehe unter Abschnitt «Private Kapitalflüsse: Wachstumstrend gebrochen»). Dieser Rückfluss verschärfte die Krise in übersteigertem Masse. Die Anleger flüchteten sich in die Qualität. Die Renditen erstklassiger Anlagen waren deshalb im vergangenen Jahr zumeist rückläufig.

Die Finanzkrise hat jedoch auch den Impuls gegeben, die notwendigen weltweiten Reformen im Finanzsektor zu beschleunigen. Makroökonomische Stabilität und Finanzstabilität müssen Hand in Hand gehen, weswegen das Bankwesen und die Finanzsysteme der Stärkung bedürfen, wie die BIZ postuliert. Einerseits braucht es besseren Zugang zu Informationen. In den letzten Jahren haben denn auch immer mehr Länder die Special Data Dissemination Standards des Internationalen Währungsfonds (IWF) übernommen. Damit sollen Krisensituationen früher und zuverlässiger erkannt werden. Der weiter verbreiteten seriösen Anwendung der Grundsätze für eine wirksame Bankenaufsicht, im Schosse der BIZ durch den Basler Ausschuss für Bankenaufsicht zusammen mit den Aufsichtsbehörden aus aufstrebenden Ländern ausgearbeitet, kommt präventive Funktion zu. Dazu gehört auch eine gewisse Standardisierung und eine grössere Transparenz der Rechnungslegung der Banken. Zur Stabilisierung der ausgebrochenen Finanzkrisen wurden schliesslich von der internationalen Staatengemeinschaft mit dem IWF an der Spitze den betroffenen Ländern umfangreiche Liquiditätshilfen gewährt. (Siehe unter Abschnitt 1.4.1. «Der Internationale Währungsfonds»). Zudem wurden in einigen Fällen Anstrengungen unternommen, auf die beteiligten internationalen Banken einzuwirken, die fällig werdenden Verbindlichkeiten umzuschulden oder zu erneuern. Die BIZ forderte, der private Sektor müsse in gewissem Umfang bereit sein, jene Schuldner, denen er in der Vergangenheit allzu leichtfertig Kredite gewährt habe, weiterhin mit Mitteln zu versorgen (NZZ, 9. Juni 1998).

Schliesslich urteilt die BIZ im Hinblick auf die teils massiven Kritiken gegenüber den IWF-Interventionen, dass die Asienkrise die erste nach dem Zweiten Weltkrieg sei, bei der die wichtigsten Internationalen Gläubiger Banken waren und die wichtigsten Schuldner dem Privatsektor angehörten. Für die Handhabung und die Bewältigung solcher Krisen gebe es keine etablierten Lösungen. Es würde wohl noch Jahre dauern, bis alle Lehren aus diesen Erfahrungen gezogen seien.

\section{BIZ-Statistiken zum internationalen Bankgeschäft}

Die Asienkrise hat gemäss Angaben der BIZ dazu geführt, dass ihren Statistiken zum internationalen Bankgeschäft wieder mehr Aufmerksamkeit geschenkt wird. Ihre Vierteljährlichen Statistiken zum internationalen Bankgeschäft gehen vom Standort der berichtenden Banken aus. Sie messen die Forderungen und Verbindlichkeiten von Geschäftsbanken mit Sitz in den 24 Ländern, die der BIZ Bericht erstatten. Das sind 18 Industrieländer und 6 weitere Finanzplätze. Diese Statistiken werden nach Art der Schuldner (Banken und Nichtbanken) sowie nach Währung der Forderung aufgeschlüsselt. Somit wer- 
den vor allem Umfang und Zusammensetzung der internationalen oder grenzüberschreitenden Kapitalströme erfasst.

Die Halbjährlichen konsolidierten Statistiken zum internationalen Bankgeschäft enthalten die Bankkredite für die verschiedenen Länder nach Fälligkeiten und Sektor sowie nach dem Herkunftsland der berichtenden Banken. Diese Statistiken enthalten Forderungen von Banken aus 18 Ländern gegenüber Entwicklungsländern, osteuropäischen Ländern sowie Ländern, die der BIZ nicht berichten.

Die BIZ bemüht sich, Qualität und Aktualität der Daten weiter zu verbessern. So wiesen die halbjährlichen Statistiken den starken Anstieg der Auslandsverschuldung der asiatischen Länder schon einige Zeit vor dem Ausbruch der Asienkrise im Jahre 1997 aus. Beispielsweise hob die BIZ die rasante Zunahme der kurzfristigen Schulden Thailands schon ab 1994 hervor.

Die BIZ-Statistiken mit ihren Gläubigerdaten können als Alternative und Ergänzung zu den WeltbankSchuldenstatistiken betrachtet werden, die auf Angaben der Schuldnerländer selbst beruhen. Sie weisen eine grössere Aktualität auf. Die Publikationsfrist beträgt fünf bis sechs Monate, während die Weltbank-Schuldnerdaten ein bis zwei Jahre zurückliegen. In einigen Pällen weist die BIZ höhere Schuldenzahlen auf als die Weltbank, was umöglicherweise darauf schliessen lässt, dass einzelne Schuldnerländer ihre Schulden nicht vollständig melden»*.

*. BIZ, Entwicklung des internationalen Bankgeschäfts und der internationalen Finanzmärkte, Basel, August 1998, S. 33ff.

\subsection{AUSSENVERSCHULDUNG}

Hauptthema der Schuldendiskussion blieb die multilaterale Schuldeninitiative für schwer verschuldete arme Länder, die sogenannte HIPC-Inititiative (Heaviliy Indebted Poor Countries). Neu diskutiert wurde, wie mit den Rückständen und dem Schuldenüberhang der sogenannten Post-Conflict-Countries ${ }^{11}$ umzugehen sei. Mit besonderen Massnahmen soll solchen Ländern dazu verholfen werden, dass sie sich auch für die HIPC-Initiative qualifizieren können.

Für die übrigen Länder konzentrierte sich die Diskussion mehr über die Frage der wachsenden Integration in die globalen (privaten) Finanzmärkte, deren Form, Fristigkeiten und Risiken. Damit verbunden ist die wachsende Bedeutung von Exportkredit- und Investitionsgarantien. Die Weltbank wies darauf hin, dass die Schulden-Export-Verhältniszahlen (Debt-Export-Ratios) der südostasiatischen Länder mit Ausnahme Indonesiens 1996 nur halb so hoch waren wie jene der lateinamerikanischen Grossschuldner ${ }^{12}$. Trotzdem blieb der Pariser Klub wo die offiziellen Schulden restrukturiert werden - nicht arbeitslos. Verbesserte Bedingungen für die Umschuldungen der ärmsten Länder sowie die Behandlung Russlands waren Hauptthemen. Auch die Umschuldung kommerzieller Schulden wurde fortgesetzt. Für einige Länder wurden die hohen Binnenschulden zu einem lastenden Problem. Die Schweiz setzte ihre bewährte und international anerkannte Schuldenpolitik fort.

\section{$\square$ Trends}

Die gesamten Schulden der Entwicklungs- und Transitionsländer erhöhten sich gemäss provisorischen Angaben der Weltbank ${ }^{13}$ um 76 Milliarden Dollar auf 2171,4 Milliarden Dollar. Davon sind 1728.5 Milliarden Dollar langfristige

11. Länder, die einen Konflikt beenden (z.B. Ex-Jugoslawien, Sudan, Rwanda, Burundi etc.). Die Weltbank hat bis zum Frühjahrestreffen 1999 einen entsprechenden Grundlagenbericht zu erarbeiten.

12. The World Bank, Global Development Finance, Fig. 2.1, aaO. S. 33.

13. The World Bank, Global Development Finance, aaO. S. 160/161. 
Schulden (Tabelle Nr. 2). In geographischer Hinsicht ist festzustellen, dass die Schulden aller Regionen stiegen. Einzig die Schulden der Länder Afrikas südlich der Sahara sanken leicht. Auch die Schulden der schwer verschuldeten ärmsten Länder konnten leicht reduziert werden.

Tabelle Nr. 2: Die Gesamtverschuldung der Entwicklungs- und Transitionsländer (in Mrd. \$)

\begin{tabular}{lrrrr}
\hline & $\mathbf{1 9 8 0}$ & $\mathbf{1 9 9 0}$ & $\mathbf{1 9 9 6}$ & $\mathbf{1 9 9 7}^{\boldsymbol{1}^{\circ}}$ \\
\hline $\begin{array}{l}\text { Langfristige Verschuldung } \\
\begin{array}{l}\text { - aus öffentlichen Quellen } \\
\text { (bi- und multilateral) }\end{array}\end{array}$ & 445 & 1168 & 1650 & 1729 \\
\hline - aus privaten Quellen & 171 & 595 & 828 & 820 \\
\hline Kurzfristige Verschuldung & 207 & 513 & 569 & 609 \\
\hline Kredite des IWF & 147 & 241 & 385 & 381 \\
\hline Gesamtverschuldung & 12 & 35 & 60 & 62 \\
\hline
\end{tabular}

Tabelle Nr. 3: Schuldenindikatoren 1996/97

\begin{tabular}{lrrrr}
\hline Ländergruppe & D/EX & D/EX & DS/EX** & DS/EX \\
\hline (in Prozenten) & 1996 & $1997 p$ & 1996 & $1997 p$ \\
\hline Alle Entwicklungsländer & 137,4 & 134,3 & 17,2 & 16,7 \\
\hline - Hochverschuldet, Einkommen tief & 361,7 & & 15,3 & \\
\hline - Hochverschuldet, Eink. mittel & 284,2 & & 36,0 & \\
\hline - Mässig verschuldet, Eink. tief & 197,2 & & 22,9 & \\
\hline - Mässig verschuldet, Eink. mittel & 116,6 & & 21,2 & \\
\hline - Andere & 79,2 & & & \\
\hline Region & & & 14,0 & 11,5 \\
\hline - Sub-Sahara Afrika & 221,6 & 201,7 & 13,0 & 11,7 \\
\hline - Ostasien, Pazifik & 101,8 & 102,6 & 22,0 & 21,0 \\
\hline - Südasien & 193,6 & 179,8 & 11,4 & 10,6 \\
\hline - Europa, Zentralasien & 104,8 & 102,0 & 32,3 & 34,1 \\
\hline - Lateinamerika, Karibik & 198,0 & 192,6 & 11,4 & 10,5 \\
\hline - Mittlerer Osten, Nordafrika & 111,4 & 114,9 & & \\
\hline
\end{tabular}

Quelle Tabellen 2 und 3: Weltbank, Global Development Finance 1998, Summary Tables, a. a. O., S. $159 \mathrm{ff}$.

P provisorische Angaben

* $\mathrm{D} / \mathrm{EX}=$ Verhältnis Schulden/Exporte von Gütern und Dienstleistungen

** DS/EX = Verhältnis Schuldendienst/Exporte von Gütern und Dienstleistungen

\section{$\square$ Die HIPC-Initiative}

Die 1996 lancierte multilaterale Initiative für die schwer verschuldeten ärmeren Länder will die Schuldenlast dieser Länder auf ein nachhaltiges Mass zurückführen und gleichzeitig soziale und ökonomische Reformen vorantreiben, damit die betroffenen Länder von fortdauernden Umschuldungen wegkommen. 41 Länder sind als HIPC klassiert, 33 davon aus Afrika und je vier aus Asien und Lateinamerika. Ihr Anteil an den Gesamtschulden beträgt knapp 12\%. Als nachhaltig werden die Schulden erachtet, wenn sie $250 \%$ der Exporte nicht übersteigen oder wenn der Schuldendienst höchstens 25\% der Exporte beträgt. Erfasst werden sämtliche Gläubigerkategorien. Um sich zu qualifizieren, muss ein Land erfolgreich Strukturanpassungsprogramme durchgeführt haben. Drei Jahre nach Beginn eines solchen Programms erreicht ein Land den Entscheidungspunkt (Decision point) und nach drei weiteren Jahren den Erfüllungspunkt (Completion point), an dem zusätzliche Entschuldungsmassnahmen vollzogen werden.

JSDW 1998, S.149ff. HIPC-Initiative. 
Seit April 1997 konnten sich sieben Länder qualifizieren, d.h. sie erreichten den Decision point, nämlich Uganda, Bolivien, Burkina Faso, Guyana, Elfenbeinküste, Moçambique und Mali. Bloss zwei erreichten bis Ende 1998 den Completion point (Tabelle Nr. 4). Benin qualifizierte sich nicht, weil die Schulden zu wenig hoch waren.

Tabelle Nr. 4: Entschuldungsmassnahmen unter der HIPC-Initiative

\begin{tabular}{lcccc}
\hline Land & $\begin{array}{c}\text { Decision } \\
\text { point }\end{array}$ & $\begin{array}{c}\text { Completion } \\
\text { point }\end{array}$ & $\begin{array}{c}\text { Entschuldung } \\
\text { nominal (Mio. \$) }\end{array}$ & $\begin{array}{c}\text { Entschuldung } \\
\text { in \% der Schulden }\end{array}$ \\
\hline Uganda & April 97 & April 98 & 650 & 20 \\
\hline Bolivien & Sept. 97 & Sept. 98 & 760 & 13 \\
\hline Burkina Faso & Sept. 97 & April 2000 & 200 & 14 \\
\hline Guyana & Dez. 97 & Anfangs 99 & 500 & 25 \\
\hline Elfenbeinküste & März 98 & März 2001 & 800 & 6 \\
\hline Mosambik & April 98 & Juni 99 & 2900 & 57 \\
\hline Mali & Sept. 98 & Dez. 99 & 250 & 10 \\
\hline Total & & & $\mathbf{6 0 6 0}$ & \\
\hline
\end{tabular}

Quelle: IMF, Debt Initiative for the Heaviliy Indepted Poor Countries, September 1998.

Bei allen sieben Ländern wurden die fixierten Dreijahresfristen zum Erreichen der Decision and Completion Points flexibel gehandhabt, d.h. gekürzt. Bei weiteren Ländern wird derzeit die Qualifikation geprüft, darunter Äthiopien, Guinea, Guinea-Bissau, Mauretanien, Niger, Senegal, Sierra Leone, Togo, Tschad, Vietnam, wobei nicht zu erwarten ist, dass alle die Anforderungen für eine Qualifizierung erreichen werden.

IWF und Weltbank überprüften kurz vor ihrer Frühjahrestagung und vor der Jahrestagung im Oktober 1998 mit einem Progress Report ${ }^{14}$. Die Exekutivräte von IWF und Weltbank verlängerten den Eintrittszeitraum bis Ende 2000. Postconflict-countries können sich allenfalls auch qualifizieren, wenn sie mit speziellen Massnahmen die HIPC-Kriterien erreichen. Diese Ausweitung hat eine Steigerung der erwartenden Gesamtkosten zur Folge, die jetzt auf 9,7 Milliarden Dollar geschätzt werden.

Die Finanzierung ist weiterhin nicht endgültig geregelt. Die Weltbank hat bislang 750 Millionen Dollar aus ihren laufenden Gewinnen in den HIPC Trust Fund überwiesen. Im Juli empfahlen die Weltbank-Exekutivdirektoren, weitere 100 Millionen aus dem Nettogewinn 1998 in den Trust Fund zu transferieren. Die Schweiz hat mit Bundesratsentscheid vom 19. August 1998 die zweite Tranche von 20 Millionen Franken für den Schweizer Beitrag in den HIPC Trust Fund der Weltbank freigegeben. Damit leistet die Schweiz insgesamt 40 Millionen Franken. Zudem ist das Bawi Hauptinitiant eines Capacity-Building Programme. Zusammen mit Dänemark, Österreich und Schweden und in Absprache mit IWF/Weltbank finanziert die Schweiz technische Hilfe für die Erarbeitung von nationalen Entschuldungsstrategien.

凹SDW 1999, siehe unter Abschnitt «Das Schweizer Entschuldungsprogramm».

Der IWF seinerseits rechnet zwar damit, derzeit für die laufende Finanzierung genügend Mittel zu haben, um seinen Anteil über die ESAF (Erweiterte Struk- 
turanpassungsfazilität) finanzieren zu können. Auf mittlere Frist ist jedoch die Finanzierung noch nicht endgültig bereinigt. Ein weiterer Bericht über die Finanzierung durch bilaterale Beiträge sowie die allfällige Inanspruchnahme von Goldreserven ist noch gegen Ende 1998 zu erwarten. Die Schweiz beteiligt sich mit 45 Millionen Franken an der Erneuerung des ESAF-Treuhandfonds, aus dem die Kosten der HIPC-Initiative gedeckt werden sollen.

IWF und Weltbank waren mit starker Kritik über Konzept und Durchführung der HIPC-Initiative konfrontiert. IWF und Weltbank luden deshalb Regierungen und Nichtregierungsorganisationen anfangs Februar zu einem Seminar nach Paris ein. Die Schweiz war durch das Bawi und die Arbeitsgemeinschaft der Hilfswerke vertreten. Kritisch beleuchtet wurde von Eurodad die Definition der Schuldennachhaltigkeit, der Einbezug der Fiskalkriterien, der schleppende zeitliche Ablauf, fehlende Interimshilfe bis zum Completion point. Armutsindikatoren sollten für die Qualifizierung miteinbezogen werden. Schuldenerleichterungen sollten ausgewiesenermassen zu besserer sozialen Entwicklung dienen ${ }^{15}$.

\section{$\square$ Umschuldung öffentlicher Schulden}

Die Mehrzahl der Abkommen zur Umschuldung von Schulden aus bilateralen öffentlichen Quellen werden im sogenannten Pariser Klub abgeschlossen. Das sind Kredite, die Regierungen andern Regierungen gewähren. Dazu kommen Exportkredite zu Marktbedingungen, die von offiziellen Institutionen der Exportrisikogarantie bei Nichtbezahlung von privaten Gläubigern übernommen worden sind. Diese offiziellen Garantien sind im Verlaufe der 90er Jahre stark ausgeweitet worden, teils wegen aggressiver Exportpromotion zahlreicher Länder, teils wegen der sich verändernden Natur der internationalen Entwicklungsfinanzierung, wie die Weltbank schreibt ${ }^{16}$. Insbesondere die verstärkte direkte Finanzierung von Infrastrukturprojekten führte zu einer Ausweitung der Verpflichtungen nationaler Exportrisikoinstitutionen. Diese Agenturen harmonisierten in den vergangenen Jahren ihre Exportkreditpolitik durch den sog. OECDKonsensus. Bemühungen seitens Nichtregierungsorganisationen, soziale und ökologische Standards mit einzubeziehen, blieben bislang erfolglos ${ }^{17}$. Es blieb lediglich bei einer vagen politischen Absichtserklärung ${ }^{18}$. Im Oktober 1998 lud die OECD Vertreter internationaler NRO zu ersten Gesprächen ein.

Als Antwort auf die wachsenden Zahlungsschwierigkeiten vieler der ärmsten Länder wurden die Bedingungen für die Umschuldungen erleichtert. Nach dem G-7 Gipfel von 1994 in Neapel kamen die Gläubigerländer überein, falls notwendig, für zwei Drittel der Umschuldungssummen konzessionelle Bedingungen zu gewähren und allenfalls nicht nur fällig werdende Schulden (flow rescheduling), sondern auch den Schuldenbestand (Stock of debt operations) mit einzubeziehen. Im Zusammenhang mit der HIPC-Initiative beschlossen die Gläubiger, von Fall zu Fall sogar bis zu $80 \%$ Schuldenreduktion zu gewähren.

凹SDW 1998, S. 151ff., Umschuldungen im Pariser Klub.

15. WB, HIPC Debt Initiative, Taking Stock and Looking Ahead, Conference Documents, The World Bank, 3. Februar 1998, Paris.

16. The World Bank, Global Development Finance, aaO. S. 58

17. Unveröffentlichter Brief von Environmental Defense Fund (USA), Eurodad (Brüssel) und Arbeitsgemeinschaft der Hilfswerke vom 16. Dezember 1997.

18. Info Bawi Nr. 2, Bern 1998. 
Multilaterale Umschuldungen im Pariser Klub sind 1997 insgesamt sieben abgeschlossen worden mit einer Gesamtsumme von 6,3 Milliarden Dollar ${ }^{19}$. Äthiopien, Madagaskar, Tanzania und die Republik Yemen erhielten eine 67 prozentige Reduktion zugesprochen, Kamerun und Guinea eine solche von 50\%. Das sind alles HIPC-Länder. Ferner wurde 1997 auch mit Jordanien umgeschuldet, jedoch nicht zu konzessionellen Bedingungen, da Jordanien als Land mit mittlerem Einkommen klassiert ist. Die Schweiz ist bei vier Ländern mit einem Betrag von insgesamt 35,9 Millionen Franken beteiligt.

1998 schuldete der Pariser Klub insgesamt 7,3 Milliarden Dollar von den folgenden acht Ländern um: Elfenbeinküste, Senegal, Rwanda, Albanien, Indonesien, Zentralafrikanische Republik, Bosnien-Herzegowina und Bolivien.

Bilaterale Umschuldungen der Schweiz sind im Jahre 1997 sechs abgeschlossen worden mit einer Gesamtsumme von 425 Millionen Franken ${ }^{20}$, nämlich mit Guinea-Conakry (2 Abkommen), Kamerun, Madagaskar, Jordanien und Russland. 383,1 Millionen Franken bilateral umgeschuldeter Fälligkeiten betreffen Forderungen aus früheren Umschuldungen, die also ein weiteres Mal konsolidiert wurden. 1998 wurden keine bilateralen Umschuldungen getätigt.

\section{$\square$ Kommerzielle Umschuldungen}

1997 sind insgesamt neun Umschuldungsabkommen zwischen den Schuldnerländern und ihren kommerziellen Gläubigerbanken abgeschlossen worden. Sie umfassen 19,1 Milliarden Dollar laufende Schulden und 6,9 Milliarden Dollar Rückstände $^{21}$. Von den Ländern mit tiefem Einkommen kaufte Togo mit Hilfe der IDA Debt Reduction Facility 46,1 Millionen Dollar zum Wert von 12,5\% zurück. Die Elfenbeinküste (6,5 Milliarden \$) und Vietnam (0,8 Milliarden \$) restrukturierten unter der Brady-Initiative. Bosnien-Herzegovina schloss mit dem Londoner Klub der Geschäftsbanken ein Umschuldungsabkommen über 1,3 Milliarden Dollar. Von den Ländern mit mittlerem Einkommen ersetzten Argentinien, Brasilien, Ekuador, Panama und Venezuela Brady Bonds mit ungesicherten Bonds. Russland schliesslich schloss mit den Geschäftsbanken im Oktober 1997 ein Umschuldungsabkommen im Ausmass von 33,0 Milliarden Dollar.

\section{$\square$ Das Schweizer Entschuldungsprogramm}

Das schweizerische Entschuldungsprogramm beruht auf vier Pfeilern:

- bilaterale Entschuldungsmassnahmen

๑ Kommerzielle Schuldenrückkäufe

- Multilaterale Massnahmen

- Komplementäre Massnahmen inkl. Technische Hilfe

Von den für Entschuldungsmassnahmen bereitgestellten 500 Millionen Franken (400 Mio. Fr. Jubiläumsfonds und 100 Mio. Fr. Rahmenkredit wirtschafts- und handelspolitische Massnahmen) sind bis Ende 1998390 Millionen verpflichtet. Es verbleibt ein Saldo von 110 Millionen Franken.

Seit 1993 hat die Schweiz bilaterale Forderungen gegenüber 28 hochverschuldeten, armen Ländern aufgekauft, dabei 19 Ländern Schulden in der Höhe von

19. The World Bank, Global Development Finance, aaO. S. 82, und ERG-Bericht 97, Bawi/ERG 1998, S. 19.

20. Aussenwirtschaftsbericht 1997, Bundesblatt vom 10. März 1998, S. 870.

21. The World Bank, Global Development Finance, aaO. S. 83/84. 
1,1 Milliarden Franken erlassen und in zwölf Ländern Gegenwertfonds in der Höhe von 268 Millionen Franken errichtet. Ende 1997 sind Guinea 10,5 Millionen Franken Schulden erlassen und ist dort ein Gegenwertfonds von 1,05 Millionen Franken errichtet worden. 1998 wurden Schulden in geringer Höhe von Jemen annulliert. Von den neun verbleibenden Ländern könnten in näherer Zukunft Kamerun, Madagaskar, Sierra Leone und Liberia entschuldbar sein.

Die Schweiz finanzierte bis Mitte 1998 kommerzielle Schuldenrückkäufe der Weltbanktochter IDA mit 77 Millionen Franken und ist damit grösste bilaterale Beitragsgeberin. Bisher profitierten elf Länder von den Beiträgen der Schweiz. Mit den Beiträgen anderer Geber konnten damit mehr als 1,8 Milliarden Dollar kommerzieller Schulden zurückgekauft und gestrichen werden. Neue Begünstigte waren seit Beginn 1997 die Elfenbeinküste, Guinea, Guyana und Mauretanien. Derzeit sind Verhandlungen im Gange, um Tansania mit kommerziellen Schuldenrückkäufen zu begünstigen.

Im vergangenen Jahr wurden hingegen keine Finanzierung von Zahlungsrückständen gegenüber den internationalen Finanzinstitutionen getätigt. Laufende Verpflichtungen gegenüber den internationalen Finanzierungsinstitutionen hat die Schweiz 1997 für Uganda und Bolivien im Vorfeld der HIPC-Initiative übernommen. 1998 beteiligte sich die Schweiz zusammen mit andern Ländern an der Finanzierung der Schulden gegenüber multilateralen Institutionen von Guinea-Bissau (vor deren Krieg) und mit 8 Millionen Franken im Falle Nicaraguas. Künftig will die Schweiz ihre Unterstützung auf diesem Gebiet soweit wie möglich über die HIPC-Initiative abwickeln. In diesem Rahmen sollen allenfalls auch HIPC-Länder unterstützt werden, die sich zwar für die HIPC-Initiative qualifizieren können, aber erst später davon profitieren werden.

$\mathrm{Zu}$ den Komplementärmassnahmen gehörte die Vergabe von Neugeld in Form von Zahlungsbilanzhilfen an ärmere Länder, die sich dank vorsichtiger Politik nicht überschuldet hatten. 1997 und 1998 sind keine derartigen Massnahmen mehr getätigt worden. Zudem leistet die Schweiz technische Hilfe im Entschuldungsbereich. Einerseits unterstützt die Schweiz weiterhin das von der $U N C$ $T A D$ durchgeführte Programm zur Informatisierung der Schuldenverwaltung. 1997 und 1998 sind vom Bawi Beiträge an Guinea-Bissau, Bolivien, Nicaragua, Peru, Jordanien, Vietnam und Burkina Faso geleistet worden. Anderseits ist sie Initiantin des HIPC Capacity Building Program, das die betroffenen Länder bei der Durchführung der unter der HIPC-Initiative notwendigen Analysen unterstützen will. Mit dabei sind auch Dänemark, Österreich und Schweden, Grossbritannien beabsichtigt, beizutreten (siehe unter «HIPC-Initiative»). Dieses Programm dient im weiteren auch als Plattform für einen regelmässigen Gedankenaustausch unter den Akteuren, die sich mit Fragen der Entschuldung und Schuldenverwaltung befassen. Letztmals tagte der entsprechende Steuerungsausschuss unter Teilnahme von Weltbank, IWF, UNDP, UNCTAD, HIPCLändern und Geberländern im Juni 1998 in Bern und im November in London.

Das Bawi verfolgt im weiteren aufmerksam weitere Themenbereiche, wie die Verschuldung gegenüber Nicht-OECD-Staaten sowie die interne Verschuldung bestimmter Länder. 


\subsection{INTERNATIONALE INVESTITIONEN}

Die internationalen Direktinvestitionen erreichten gemäss UNCTAD 1997 eine neue Rekordhöhe. Triebkräfte waren die Liberalisierungsanstrengungen im Handel und bei den Investitionen sowie die Privatisierung. Die Zielländer erhoffen sich nicht nur einen Kapitalzustrom, sondern vermehrten Zugang zu Technologie und Wissen. Die Verhandlungen im Rahmen der OECD um das Multilaterale Investitionsabkommen wurden anfangs Dezember 1998 ergebnislos abgebrochen.

\section{$\square$ Der UNCTAD-Weltinvestitionsbericht}

Der weltweite Bestand ausländischer Direktinvestitionen belief sich gemäss dem jährlich erscheinenden UNCTAD-Weltinvestitionsbericht 1998 auf rund 3500 Milliarden Dollar, 13\% mehr als im Vorjahr ${ }^{22} .2350$ Milliarden sind in Industrieländern getätigt worden, 1050 Milliarden in Entwicklungs- und Transitionsländern.

Von den neuen, im Jahre 1997 zufliessenden 400 Milliarden Direktinvestitionen landeten 233 Milliarden in den Industriestaaten, und 167 Milliarden Dollar flossen in die Entwicklungs- und Transitionsländer (Tabelle Nr. 5).

Nach wie vor sind bloss wenige Entwicklungsländer Hauptnutzniesser des Investitionszuflusses. Zu den grössten Zuflussländern im Süden und Osten gehörten: China (41 Mrd. \$), Brasilien (11 Mrd.), Singapur (9 Mrd.), Mexiko (8 Mrd.), Indonesien (6 Mrd.), Argentinien (5 Mrd.), Malaysia (5 Mrd.), Polen (5 Mrd.), Chile (4 Mrd.) und Peru (4 Mrd.).

Noch einseitiger ist dieses Bild, wenn man die Herkunft der Direktinvestitionen betrachtet. Über $90 \%$ der ausländischen Direktinvestitionen stammen aus Industrieländern, bloss knapp 10\% aus Entwicklungs- und Transitionsländern. Dieser Anteil hat sich allerdings seit Beginn der 90er Jahre mehr als verdoppelt.

Tabelle Nr. 5: Ausländische Direktinvestitionen nach Regionen und Ländern, 1995-1997

\begin{tabular}{lcccccr}
\hline \multirow{2}{*}{ Region/Land } & \multicolumn{3}{c}{ Zufliessende DI* } & \multicolumn{3}{c}{ Abfliessende DI* } \\
& $\mathbf{1 9 9 5}$ & $\mathbf{1 9 9 6}$ & $\mathbf{1 9 9 7}$ & $\mathbf{1 9 9 5}$ & $\mathbf{1 9 9 6}$ & $\mathbf{1 9 9 7}$ \\
\hline Industrieländer & 211 & 195 & 233 & 306 & 283 & 359 \\
\hline Europa & 123 & 100 & 115 & 174 & 168 & 196 \\
\hline Nordamerika & 70 & 83 & 99 & 103 & 83 & 128 \\
\hline Japan & 0,0 & 0,2 & 3,2 & 23 & 23 & 26 \\
\hline Entwicklungsländer & 106 & 130 & 149 & 46 & 49 & 61 \\
\hline Afrika & 5,1 & 4,8 & 4,7 & 0,5 & 0,3 & 1,1 \\
\hline Lateinamerika/Karibik & 32 & 44 & 56 & 2,3 & 2,3 & 9,1 \\
\hline Asien & 67 & 80 & 87 & 42,6 & 46,5 & 50,6 \\
\hline davon Südostasien & 67 & 77 & 82 & 41,8 & 47,3 & 50,2 \\
\hline Osteuropa & 14 & 12 & 18 & 0,5 & 1,0 & 3,3 \\
\hline Total & $\mathbf{3 3 1}$ & $\mathbf{3 3 8}$ & $\mathbf{4 0 0}$ & $\mathbf{3 5 3}$ & $\mathbf{3 3 4}$ & $\mathbf{4 2 4}$ \\
\hline
\end{tabular}

Quelle: UNCTAD, World Investment Report 1998, Table IB.1/B.2 (1997: provisorische Zahlen).

${ }^{*}$ DI: Direktinvestitionen

Die Finanzkrise Asiens hat sich in den UNCTAD-Zahlen noch nicht niedergeschlagen. Die UNCTAD erwartet jedoch, dass die Direktinvestitionen 1998 in 
den asiatischen Ländern bestenfalls stagnieren, während für Lateinamerika und die Karibik weitere Steigerungen erwartet werden. Der Bericht erläutert zudem eingehend, warum ausländische Direktinvestitionen deutlich weniger schwanken (weniger volatil sind) als Portfolioinvestitionen.

Die UNCTAD stellt in ihrem Bericht eine rasant steigende Zahl grenzüberschreitender Firmenkäufe durch ausländische transnationale Unternehmungen fest. Dazu gehören auch Käufe privatisierter Staatsunternehmen. Mit einher geht damit auch eine grössere Konzentration des Besitzes. Weiter konstatiert die UNCTAD eine wachsende Zahl grenzüberschreitender Abkommen zwischen zwei Firmen, wobei sich die Mehrzahl dieser Zusammenarbeitsverträge auf Technologie beziehen.

\section{$\square$ Direktinvestition in und aus der Schweiz}

Der UNCTAD World Investment Report weist für 1997 einen Bestand von 56,5 Milliarden Dollar an ausländischen Direktinvestitionen in der Schweiz aus. Der Zufluss betrug 3,5 Milliarden und war fast gleich hoch wie im Vorjahr. Umgekehrt belaufen sich die schweizerischen Direktinvestitionen im Ausland auf 156,7 Milliarden Dollar, wobei im letzten Jahr 12 Milliarden hinzugekommen sind. Der Bestand schweizerischer Direktinvestitionen im Ausland beträgt 49,2\% des Bruttosozialproduktes, ein Wert, den kein anderes Land auch nur annähernd erreicht.

Die Schweizerische Nationalbank weist provisorisch für 1997 Schweizer Direktinvestitionen in die Schwellenländer im Ausmass von 726 Millionen Franken aus. Im Vorjahr waren es noch 1406 Millionen gewesen ${ }^{23}$. Dabei fiel vor allem ins Gewicht, dass einerseits Schweizer Investoren in Brasilien massiv desinvestierten (-329 Mio. Fr.). Anderseits wirkte sich die Finanzkrise der südostasiatischen Länder bereits aus. In Südkorea wurde ebenfalls desinvestiert (-61 Mio. Fr.), während die Investitionsströme nach Malaysia, Singapur und Thailand deutlich kleiner waren als im Vorjahr (Tabelle Nr. 6). Der Bestand schweizerischer Investitionen in Schwellenländern belief sich Ende 1997 somit auf 15'452 Millionen Franken.

Tabelle Nr. 6: Schweizerische Direktinvestitionen

in Entwicklungs- und Schwellenländern, 1995-97 (in Mio. Fr.)

\begin{tabular}{lrrrrrr}
\hline \multirow{2}{*}{ Region } & \multicolumn{3}{c}{ Kapitalbestand } & \multicolumn{3}{c}{ Kapitalexport } \\
\hline Schwellenländer & $\mathbf{1 9 9 5}$ & $\mathbf{1 9 9 6}$ & $\mathbf{1 9 9 7}$ & $\mathbf{1 9 9 5}$ & $\mathbf{1 9 9 6}$ & $\mathbf{1 9 9 7}^{\mathrm{p}}$ \\
\hline Lateinamerika & 11863 & 14044 & 15542 & 55 & 1406 & 716 \\
\hline Asien & 7216 & 8528 & 8796 & 186 & 260 & -225 \\
\hline Entwicklungsländer & 4647 & 5516 & 6656 & -242 & 1146 & 951 \\
\hline Lateinamerika & 19318 & 21393 & 28691 & 487 & 1057 & 1716 \\
\hline Afrika & 16524 & 17218 & 23329 & 2 & -267 & 839 \\
\hline Asien & 1178 & 1290 & 1626 & 25 & 90 & 230 \\
\hline
\end{tabular}

Quelle: Schweiz. Nationalbank, 4. Quartalsbericht 1998.

${ }^{\mathrm{P}}$ provisorische Angaben. 
Hingegen wuchsen die schweizerischen Direktinvestitionen in die Entwicklungsländer erneut stark an. Insgesamt erreichten sie 1716 Millionen Franken, gegenüber 1057 Millionen im Vorjahr. Dennoch erreichten sie die Rekordhöhe von 1991 von 2056 Millionen nicht. Ende 1997 betrug der Kapitalbestand in Entwicklungsländern mittlerweile 28'691 Millionen Franken, 34\% mehr als im Vorjahr.

Die Direktinvestitionen aus Schwellenländern in der Schweiz bildeten sich 1997 um 65 Millionen Franken zurück auf einen Bestand von noch 56 Millionen. Entwicklungsländer hatten bis Ende 1997 insgesamt 1292 Millionen Franken an Direktinvestitionen in der Schweiz, 78 Millionen mehr als im Vorjahr.

\section{$\square$ Bilaterale Investitionsverträge}

Die Zahl der bilateralen Investitionsverträge ist bis Ende 1997 auf über 1500 angestiegen, gegenüber 1330 am Ende des Vorjahres. Die meisten davon regeln Investitionsbeziehungen von Industrieländern mit Entwicklungs- und Transitionsländern. Für die ersteren ist es ein Absicherungsinstrument für ihre Direktinvestitionen sowie eine Erleichterung für die konkrete Geschäftstätigkeit, für die zweiten ein Hilfsmittel, Investitionen anzuziehen. Mehr und mehr sind auch regional gültige Abkommen geschlossen worden. Die UNCTAD kommt dennoch zum Schluss, dass diese Abkommen weit weniger Investitionsentscheide beeinflussen als Marktgrösse, Wachstumserwartungen, Wechselkursverhältnisse und Länderrisiken.

Trotzdem glaubt die UNCTAD, multilateral geltende Regeln könnten die internationale Investitionstätigkeit anregen, sofern die Regeln den Investoren grössere Sicherheiten und den Empfängerländern grössere Stabilität, Voraussehbarkeit und Transparenz gewähren würden.

\section{$\square$ Das Multilaterale Investitionsabkommen (MAI)}

Ist das Multilaterale Investitionsabkommen (MAI) mit dem Rückzug Frankreichs aus den entsprechenden OECD-Verhandlungen auf unbestimmte Zeit eingefroren? Oder werden die Verhandlungen bei der WTO fortgesetzt? Erhält die UNCTAD eine neue Chance? Oder gibt es überhaupt kein Multilaterales Investitionsabkommen?

Die vielen Fragezeichen verdeutlichen, wie verworren die Situation um das sehr umstrittene Anliegen multilateraler Investitionsregeln ist. Tatsache bleibt, dass die OECD seit 1995 Gespräche führt über multilaterale Investitionsregeln und ein entsprechendes Abkommen vorbereitet hat.

Hintergrund ist die Tatsache, dass internationale Regeln für grenzüberschreitende Investitionen fehlen. Dies, obwohl internationale Investitionen doppelt so schnell wachsen wie der Welthandel. Nationale wie regionale Regeln offenbaren widersprüchliche Tendenzen. Einerseits führen Nationalstaaten immer wieder protektionistische Massnahmen ein, um Unternehmen ausländischer Herkunft zu benachteiligen. Anderseits locken Nationalstaaten ausländische Investitionen mit Subventionen aller Art und oft auch unter Missachtung bestehender Umwelt- und Sozialnormen im verschärften Standortwettbewerb an.

Die OECD-Länder begannen 1995 mit Verhandlungen, mit der Idee, ein von der OECD unabhängiges Regelwerk zu schaffen, das dann nicht nur OECD-Ländern, sondern allen interessierten Staaten offen stehen würde. Aber zu den kon- 
kreten Verhandlungen wurden einzelne Länder des Südens und Ostens erst zu einem späteren Zeitpunkt als Beobachter eingeladen.

Zentraler Grundsatz war stets die Nichtdiskriminierung, d.h. die Gleichbehandlung inländischer wie ausländischer Investoren sowie das Prinzip der Drittländermeistbegünstigung. Weiter gehörten Regeln über den freien Transfer von Gewinnen und Kapital sowie über faire Entschädigung bei Enteignungen zum vorgesehenen Abkommen. Nationale Ausnahmen waren vorgesehen. Umweltund Sozialnormen sollten mit berücksichtigt werden ${ }^{24}$. Ein vorgesehenes Streitschlichtungsverfahren privilegierte die Investoren.

Die Verhandlungen erfolgten vorerst weitgehend abgeschirmt von der Öffentlichkeit. Sobald jedoch mehr Einzelheiten bekannt wurden, verstärkte sich weltweit der Widerstand gegen ein solches Abkommen bis hin zur totalen Ablehnung. Umwelt-, Kultur- und Konsumorganisationen wandten sich dagegen, gleich wie Gewerkschaften und Entwicklungsorganisationen. Hauptkritikpunkte waren die Übertragung staatlicher Regelungskompetenzen an internationale Investoren sowie die Errichtung internationaler Schiedsverfahren. Das bisher unbekannte Thema wurde zu einem Reisser in den Medien. Widersprüche zwischen nationalen Interessen, etwa zwischen Frankreich und den USA, wurden unüberbrückbar. Im Oktober 1998 zog sich Frankreich aus den Verhandlungen zurück. Sie wurden auf niedriger Stufe fortgesetzt. Weitere Verhandlungen Anfangs Dezember 1998 wurden ergebnislos abgebrochen ${ }^{25}$.

Die offizielle Schweiz vertrat stets den Standpunkt, ein multilaterales Regelwerk würde den schweizerischen Interessen am besten dienen. Das federführende Bawi setzte sich sogar für den Einbezug von bestehenden Umwelt- und Sozialregeln ein. Die kritischen Vorbehalte in der Schweiz kamen von der Arbeitsgemeinschaft der Hilfswerke, der Erklärung von Bern und von den Umweltorganisationen ${ }^{26}$. Kleinere, radikalere Organisationen, hauptsächlich aus Genf, erklärten gar den totalen Widerstand. Im Parlament wurden mehrere Vorstösse eingereicht.

Die Haltung des Bundesrates kann etwa wie folgt umrissen werden: multilaterale Regeln

๑ erhöhen die internationale Rechtssicherheit,

口 dämmen den wilden Standortwettbewerb ein,

口 schränken die Macht der grossen Länder ein,

- erlauben den Einbezug von Sozial- und Umweltstandards.

Demgegenüber verlangten die Nichtregierungsorganisationen unter anderem den Einbezug der Entwicklungsländer in die Verhandlungen, die Beschränkung auf Direktinvestitionen, wettbewerbspolitische Regeln, Gleichgewicht zwischen Rechten und Pflichten für die Investoren usw.

24. Vgl. dazu OECD Policy Brief Nr. 2/1997, OECD Paris, sowie: Marino Baldi, Das Investitionsabkommen (MAI), in: Die Volkswirtschaft, Magazin für WirtschafsPolitik, 12/97, Bern 1997.

25. Abbruch der MAI-Verhandlungen, NZZ, 5./6. Dezember 1998

26. Gerster, Richard, Multilaterales Abkommen zu Investitionen (MAI) und Nachhaltige Entwicklung: Verbindliche Leitplanken erforderlich, in: Die Volkswirtschaft aaO. Nr. 1/98. Bosshard, Peter, und Sekinger Urs, MAI - Freipass für Multinationale Konzerne? EvB, Solifonds, Greenpeace, Zürich, April 1998. 


\subsection{DIE INTERNATIONALEN FINANZIERUNGSINSTITUTIONEN}

Der Internationale Währungsfonds (IWF) und die Weltbank waren durch die Finanzkrise einer besonderen Herausforderung gegenübergestellt. Beide Institutionen sahen sich massiver Kritik gegenüber. Die Zusammenarbeit und die vereinbarte Arbeitsteilung zwischen beiden Instituten war einer harten Belastungsprobe ausgesetzt. Sowohl IWF und Weltbank erhöhten ihre Ausleihungen in markanter Weise.

\subsubsection{DER INTERNATIONALE WÄHRUNGSFONDS}

Im Geschäftsjahr 1997/98, das am 30. April 1998 endete, machte der IWF infolge der Finanzkrise um sechsfach höhere Kreditzusagen als im Vorjahr. Auch die effektiven Auszahlungen überstiegen die Vorjahreszahlen um mehr als das Dreifache. Das schaffte dem IWF Liquiditätsprobleme, zusätzlich noch erschwert durch die von den USA verzögerte Zustimmung zur vereinbarten Kapitalerhöhung. Die Stossrichtung des IWF in der Finanzkrise provozierte heftige Debatten.

\section{$\square$ Rekordhohe Kreditzusagen}

Die Finanzkrise Asiens und Russlands hatte einschneidende Folgen für die finanziellen Operationen des IWF. Insgesamt gewährte der IWF Kreditzusagen von 31,8 Milliarden Sonderziehungsrechte (SZR) oder umgerechnet rund 42,8 Milliarden Dollar, das Sechsfache des Vorjahresbetrages ${ }^{27}$. Die Auszahlungen beliefen sich total auf 19,9 Milliarden SZR, fast das Vierfache des Vorjahres (Tabelle Nr. 7). Insgesamt vereinbarte der IWF neun Bereitschaftskredite über 27,3 Milliarden SZR, davon allein 26,7 Milliarden SZR mit den südostasiatischen Krisenländer Südkorea, Indonesien, Thailand und den Philippinen. Im weiteren schloss der IWF vier Abkommen mit der Erweiterten Fondsfazilität (2,8 Mrd. SZR) und acht neue ESAF-Kredite (1,7 Mrd. SZR) ab. Ende April waren 14 Bereitschaftsabkommen, 13 erweiterte Abkommen und 33 ESAF-Abkommen in Kraft. Die grössten Kredite bezogen Korea (11,2 Mrd. SZR), Indonesien (2,2 Mrd. SZR), Thailand (2,0 Mrd. SZR) und Russland (1,5 Mrd. SZR).

Tabelle Nr. 7: Kreditvergabe des IWF, 1995-1998 (in Mrd. SZR, jeweils Abschluss per 30. 4.)

\begin{tabular}{lrrrr}
\hline & $\mathbf{1 9 9 5}$ & $\mathbf{1 9 9 6}$ & $\mathbf{1 9 9 7}$ & $\mathbf{1 9 9 8}$ \\
\hline Auszahlungen & 11,2 & 12,3 & 5,6 & 19,9 \\
\hline - Afrika & 1,0 & 2,3 & 1,0 & 0,9 \\
\hline - Asien & 0,4 & 0,4 & 0,2 & 16,4 \\
\hline - Europa & 2,9 & 5,2 & 3,4 & 2,2 \\
\hline - Naher Osten & 0,1 & 0,1 & 0,2 & 0,1 \\
\hline - Lateinamerika & 6,8 & 4,4 & 0,9 & 0,3 \\
\hline Rückzahlungen & 4,2 & 7,1 & 7,2 & 4,4 \\
\hline Nettotransfer & 7,0 & 5,2 & $-1,6$ & 15,5 \\
\hline Ausstehende Kredite & 36,8 & 42,0 & 40,5 & 56,0 \\
\hline Überfällige Verpflichtungen & 3,0 & 2,2 & 2,2 & 2,3 \\
\hline Zahl der Kreditnehmer & 99,0 & 97,0 & 95,0 & 94,0 \\
\hline
\end{tabular}

Quelle: IMF Annual Report 1998, Tabelle 12 und 13.

27. IMF, Annual Report Financial Year 1997/98, Washington 1998. 
Im Dezember 1997 schuf der Exekutivrat die Zusätzliche Reservefazilität (Supplemental Reserve Facility), um damit den Ländern mit ausserordentlichen Zahlungsbilanzschwierigkeiten rasche und zusätzliche Mittel zur Verfügung stellen zu können.

Die ausserordentlich hohe Mittelbeanspruchung führte zu einer deutlichen Verringerung der frei verfügbaren Mittel des IWF von 43,5 Mrd. SZR im Vorjahr auf nur noch 22,6 Mrd. SZR.

\section{Stärkung der Eigenmittel}

Die im letzten Jahr grundsätzlich beschlossene Quotenerhöhung um $45 \%$ auf neu 212 Milliarden SZR konnte während des Geschäftsjahres noch nicht unter Dach gebracht werden. Der US-amerikanische Kongress lehnte es mit einer Koalition sowohl von konservativen wie ökologischen Abgeordneten lange Zeit ab, die erforderlichen Mittel zu sprechen. Erst Mitte Oktober 1998 einigte sich die republikanische Kongressmehrheit in einer Gesamtbudgetausmarchung mit Präsident Clinton, die benötigten Mittel für die Kapitalaufstockung des IWF grundsätzlich $\mathrm{zu}$ bewilligen. Zur effektiven Quotenerhöhung bedarf es der Zustimmung von drei Fünfteln der Mitgliedsländer mit mindestens $85 \%$ der Gesamtsumme, was ohne US-Beitrag nicht möglich ist.

Die Quote der Schweiz geht mit dieser Quotenerhöhung von 1,69 auf 1,63\% zurück, jene der Schweizer Stimmrechtsgruppe von 2,7 auf 2,6\%. Dies ist die Folge einer Anpassung der Quote verschiedener Länder an ihre effektive weltwirtschaftliche Bedeutung. Der Bundesrat bewilligte am 8. Juni 1998 die Quotenerhöhung. Die Erklärung von Bern bezichtigte in einem Brief an Bundesrat Villiger, damit habe der Bundesrat die notwendige vorherige Information des Parlamentes umgangen. Bundesrat Villiger bezeichnete diesen Vorwurf in seinem Antwortschreiben als nicht haltbar. Nationalrat Remo Gysin (SP, BS) reichte in der Folge eine parlamentarische Initiative ein, wonach künftig Kapitalaufstockungen des IWF vom Parlament zu genehmigen seien ${ }^{28}$.

Nachdem der Ständerat schon in der Herbstsession 1997 dem Beitritt der Schweiz zur Neuen Kreditvereinbarung (NKV, engl. NAB) zugestimmt hatte, folgte ihm der Nationalrat in der Dezembersession 1997. An der NKV nehmen 25 Länder teil, darunter auch alle elf Länder, die schon die Allgemeine Kreditvereinbarung $(A K V$, egl. $G A B)$ abgeschlossen haben.

凹 JSDW 1998, S.159, New Arrangement to Borrow (NAB).

Die AKV wurde im Juli 1998 zugunsten Russlands in Anspruch genommen. Drei Viertel des damaligen Russlandkredites wurden mit der AKV finanziert. Die Schweizerische Nationalbank leistete einen Beitrag von 378 Millionen SZR (ca. 750 Mio. Fr.).

\section{$\square$ Die Überwachungstätigkeit}

Die schon im Vorjahr verstärkte Überwachungstätigkeit erfuhr infolge der Finanzkrise eine weitere Intensivierung. Traditionelles Instrument bleibt die sog. Art.-IV-Konsultation, mit welcher der IWF die Wirtschaftspolitik seiner

28. Peter Bosshard, Alte Rezepte für neue Krisen. Die Rolle des Internationalen Währungsfonds in Ostasien, die Kapitalerhöhung des IWF und die Schweiz, Erklärung von Bern, August 1998. Brief EvB vom 4. Juni 1998 an Bundesrat Villiger vom 1. Juli 1998, Parlamentarische Initiative Gysin Remo vom 17. Juni 1998. 
Mitgliedsländer in der Regel jährlich überprüft. Der IWF verstärkte im Gefolge der Finanzkrise seine Überwachungstätigkeit. Schwergewicht wurde aber auch auf die Förderung guter Regierungstätigkeit (Good Governance), die Verstärkung der Wirtschaftsdaten, die Verbesserung der Transparenz in Fiskalfragen gelegt. Der IWF erliess entsprechende Richtlinien. Seine eigene Transparenz erhöhte der IWF, indem er fortan detaillierte Berichte über die erfolgten Konsultationen gemäss Art. IV publiziert. Der IWF ermutigt seine Mitgliedsländer, die Letters of Intent und die Policy Framework Papers zu veröffentlichen. Selber veröffentlichte der IWF die HIPC-Länderpapiere und zahlreiche weitere Dokumente, die bislang nicht zugänglich waren.

Jeweilen im November besucht eine IWF-Delegation die Schweiz und überprüft im Rahmen der Art.-IV-Konsultation deren Wirtschafts- und Geldpolitik. Neben ausgedehnten Gesprächen mit den betroffenen Departementen und der Nationalbank sowie Wirtschaftskreisen trifft die IWF-Delegation auch eine Vertretung der Arbeitsgemeinschaft der Hilfswerke. 1997 brachte die Arbeitsgemeinschaft die Frage der ökologischen Steuerreform in die Diskussion. 1998 forderte die Arbeitsgemeinschaft, die Schweiz solle eine aktivere Haltung zur Bekämpfung der Finanzkrise einnehmen. In Frage kämen gezielte Massnahmen in den von der Krise am meisten betroffenen Staaten zugunsten kleiner Unternehmungen und für den Ausbau sozialer Sicherheitsnetze. Importe aus diesen Ländern sollten gefördert werden.

\section{$\square$ Technische Hilfe}

In verflossenen Jahr leistete der IWF technische Hilfe im Ausmass von annähernd 300 Personenjahren. Das kostete 17\% der gesamten administrativen Ausgaben des IWF. Der IWF leistet in folgenden Bereichen technische Hilfe (Reihenfolge in abnehmender Bedeutung): Geldpolitik und Wechselkursfragen, Fiskalfragen, Statistik, Rechtsfragen.

Die Schweiz schloss am 15. April 1998 mit dem IWF ein Abkommen zur Schaffung eines 2,5-Millionen-Dollar-Fonds für technische Hilfe des IWF zugunsten der zentralasiatischen Länder in der von der Schweiz angeführten Stimmrechtsgruppe. Die Mittel stammen aus dem $\mathrm{Bawi}^{29}$.

\section{$\square E S A F$}

Die Erweiterte Strukturanpassungsfazilität (ESAF), das zinsgünstige Fenster des IWF für die ärmsten Länder, führte in zweierlei Hinsicht zu Diskussionen. Einerseits ist die 1996 beschlossene Zwischenfinanzierung noch nicht unter Dach. Der Exekutivrat hatte dem Interimsausschuss sowohl auf das Frühjahrestreffen wie auch auf das Jahrestreffen 1998 Zwischenberichte zu erstatten. Die erforderlichen bilateralen Beiträge sind nach wie vor noch nicht zugesichert. Bis Ende Juli hatten lediglich sechs Länder Beiträge an den ESAF-HIPC Trust endgültig beschlossen. (Siehe unter «HIPC-Initiative»). Der Schweizer Beitrag, im März vom Nationalrat als Zweitrat bestätigt, ist in diesen sechs Ländern noch nicht eingeschlossen.

Anderseits hatte der Exekutivrat im Juni 1997 eine umfassende Evaluation der ESAF durch interne und externe Fachleute beschlossen. Beide Berichte sind 
nach anfänglichem Hin und Her vom IWF veröffentlicht worden und haben zu lebhaften Auseinandersetzungen geführt. Der Bericht der internen Evaluatoren ${ }^{30}$ plädierte insbesondere für bessere fiskalische Anpassungsmassnahmen, stärkere Inflationsbekämpfung und weiterführende strukturelle Reformen. Damit blieb er weitgehend im traditionellen IWF-Diskurs stecken. Die externen Evaluatoren forderten eine weitergehende enge Zusammenarbeit mit der Weltbank in sozialen Belangen in der Verbindung zwischen Makro- und Mikroökonomie und in der kurz- und längerfristigen Analyse. Die Frage wurde aufgeworfen, ob die ESAF Post-Stabilisationsphase Unterstützungsaufgaben zukommen müsste. Die externen Evaluatoren plädierten für den Einbezug alternativer Politikentwürfe und für verstärkte Ownership der betroffenen Länder ${ }^{31}$.

\section{$\square$ Kapitalverkehrsliberalisierung}

Mit dem Beschluss des Interimskomitees an der Jahresversammlung in Hongkong im Herbst 1997 hat der IWF grundsätzlich die Aufgabe, sich mit der Liberalisierung des Kapitalverkehrs zu beschäftigen. Daran hält der IWF trotz Finanzkrise fest. Die IWF-Satzungen sind allerdings noch nicht entsprechend angepasst worden.

Eine Kapitalverkehrsliberalisierung sei jedoch sehr riskant, wenn nicht vorher bestimmte Voraussetzungen erfüllt seien, liess Stanley Fisher, Stellvertretender Geschäftsführender Direktor, mehrfach wissen. Dazu gehören u.a. eine konsistente makroökonomische Politik ohne übergrosse Zahlungsbilanz-Ungleichgewichte, eine gut ausgebaute Überwachung des Finanzsystems. Der Weg der Liberalisierung müsse «geordnet» sein, mit der richtigen Reihenfolge und der zeitlichen Abstimmung der einzelnen Schritte.

Zahlreiche Kritiker verlangen im Gefolge der Finanzkrise allerdings, dass insbesondere die kurzfristigen Finanzflüsse Kontrollen und Einschränkungen unterworfen werden müssten.

\section{$\square$ Verbesserte Architektur des Internationalen Finanzsystems}

Schon nach der Finanzkrise Mexikos in den Jahren 1994-95 hatte der IWF verschiedene Initiativen ergriffen, um das internationale Finanzsystem zu stärken. Im April 1998 identifizierte der Exekutivrat eine Serie weiterer Anstrengungen als Lehren aus der Finanzkrise Asiens. Das Interimskomitee stimmte diesen während der Frühjahrestagung im April ebenfalls zu. Sie umfassen folgende Punkte:

口 Verstärkung der internationalen und lokalen Finanzsysteme. Darunter fallen verbesserte Überwachungs- und Regulierungsinstitutionen, erhöhte Unabhängigkeit der Zentralbanken, gute Regierungsführung, internationale Standards Verhaltenskodices.

口 Verstärkte Überwachungstätigkeit des IWF, insbesondere im Finanzsektor, und enge Zusammenarbeit mit Weltbank, BIZ und weiteren internationalen Organisationen.

- Erhöhte Transparenz, indem qualitativ bessere Wirtschaftsdaten rascher verfügbar gemacht werden.

30. IMF, The ESAF at Ten Years. Economic Adjustment and Reform in Low-Income Countries. Occasional Paper 156, Washington DC 1997.

31. IMF, External Evaluation of the ESAF. Report by a Group of Independent Experts. Washington DC, 24. Juni 1998. 
๑ Bestätigung der führenden Rolle des IWF im Krisenmanagement.

- Stärkeres Einbinden des privaten Sektors in die Krisenbewältigung, z.B. mittels Fristverlängerungen und Umschuldungen.

Finanzminister und Notenbankgouverneure der G-7-Länder, ergänzt mit 15 weiteren wirtschaftlich bedeutsamen Entwicklungs- und Transitionsländern aus allen Kontinenten, setzten im April 1998 drei Arbeitsgruppen ein (Internationale Finanzkrise; Transparenz und Verantwortlichkeit; Verbesserung des Finanzsystems). Die Schweiz war nicht Mitglied dieser Arbeitsgruppen und hat brieflich bei den einladenden USA protestiert. Zusammen mit Holland, Belgien und Schweden wurde die Schweiz dann immerhin zur einer weiteren Orientierungssitzung eingeladen. Die umfangreichen Berichte der Arbeitsgruppen veröffentlichte der IWF anfangs Oktober auf dem Internet und hiess Kommentare willkommen $^{32}$.

\section{$\square$ Der IWF in der Finanzkrise}

Die Finanzkrise beanspruchte nicht nur umfangreiche finanzielle Mittel des IWF (siehe unter «Rekordhohe Kreditzusagen»), sondern absorbierte auch sehr viel Arbeitszeit des Managements und des Exekutivrates. Zudem äusserten sich die Verantwortlichen in zahlreichen Reden zum Thema, und es erscheint in vielen Publikationen. Zusammengefasst kann die angemessene Politikantwort des IWF auf die Finanzkrise umrissen werden:

口 Einheimischen wie ausländischen Investoren muss glaubhaft versichert werden, dass das makroökonomische Gleichgewicht wiederhergestellt wird.

a Fiskalische Anpassungsmassnahmen sind notwendig, dürfen aber die Gesamtnachfrage nicht allzu stark kompromittieren. Insbesondere müssen die sozialen Kosten aufgefangen werden und die Restrukturierung des Finanzsystems gewährleistet sein.

口 Die Geldpolitik muss fest bleiben, um übermässige Abwertungen mit harten Inflationsfolgen und Kapitalabfluss zu minimieren.

- Strukturelle Defizite im Finanzsektor der einzelnen Ländern müssen raschmöglichst behoben werden.

口 Die gute Regierungsführung muss gewährleistet werden.

Hauptpunkte der vielfältigen Kritik waren die zu starke Eindämmung der Gesamtnachfrage über Budgetrestriktionen und Hochzinspolitik, die in den betroffenen Ländern eine viel zu starke rezessive Auswirkung gezeitigt hätte ${ }^{33}$.

In der Zwischenzeit erlaubt der IWF den betroffenen Ländern grössere Budgetdefizite und angesichts wieder erstarkender Währungsreserven auch eine weniger strenge Zinspolitik..

[ JSDW 1999, siehe unter «Die Finanzkrise».

\subsubsection{DIE WELTBANK}

\section{$\square$ Zur Geschäftstätigkeit}

Im abgelaufenen Geschäftsjahr (1. Juli 1997 bis 30. Juni 1998) hat die Weltbank die höchsten Kreditbeiträge seit ihrer Gründung zugesagt, nämlich 21,1 Millio-

32. IMF, Reports on the International Financial Architecture, Washington 1998.

33. Vgl. dazu mosquito, Globale Enwicklungsfinanzierung, Nr. 5, August 1998. 
nen Dollar für 151 Projekte seitens der IBRD und 7,5 Milliarden Dollar für 135 Projekte seitens der IDA. Dazu haben sowohl die Finanzkrise wie die grösseren Bedürfnisse vor allem Afrikas beigetragen.

Die Auszahlungen der Bank erreichten insgesamt 25,5 Milliarden Dollar, nämlich 19,8 Milliarden für die IBRD und 5,6 Milliarden für die IDA (vgl. Tabelle Nr. 8).

Der Reingewinn liegt mit 1,234 Milliarden Dollar faktisch gleich hoch wie im Vorjahr. Das täuscht nicht über die Tatsache hinweg, dass längerfristig sinkende Gewinne erwartet werden. Das dürfte zu einem Interessenkonflikt führen zwischen drei verschiedenen Gruppen: den Industrieländern, den Ländern mit mittleren Einkommen und den ärmsten Ländern. Tendenziell möchte ein Teil der Industrieländer selber weniger Beiträge zahlen und dafür mehr die Nettogewinne benutzen (z.B. für die MIGA-Kapitalerhöhung). Die Länder mit mittlerem Einkommen möchten weniger hohe Zinsen für ihre Kredite bezahlen, was den Weltbankgewinn schmälert. Und die ärmsten Länder treten für die Finanzierung spezieller Aufgaben aus dem Reingewinn ein (z.B. HIPC-Initiative).

Tabelle Nr. 8: Ressourcenfluss Weltbank 1995-1998

in Mrd. \$, Abschluss des Geschäftsjahres jeweils 30. Juni.

\begin{tabular}{lrrrr}
\hline & $\mathbf{1 9 9 5}$ & $\mathbf{1 9 9 6}$ & $\mathbf{1 9 9 7}$ & $\mathbf{1 9 9 8}$ \\
\hline Weltbank & & & & \\
\hline Kreditzusagen & 16,9 & 14,7 & 13,4 & 21,1 \\
\hline Auszahlungen & 12,7 & 13,4 & 14,0 & 19,2 \\
\hline Nettoauszahlungen & 0,9 & 1,2 & 2,1 & \\
\hline Ausstehende Kredite & 123,5 & 164,8 & 157,4 & 157,6 \\
\hline IDA & & & & \\
\hline Kreditzusagen & 5,7 & 6,9 & 4,6 & 7,5 \\
\hline Auszahlungen & 5,7 & 5,9 & 6,0 & 5,6 \\
\hline Nettoauszahlungen & 5,2 & 5,3 & 5,2 & 4,8 \\
\hline
\end{tabular}

Quelle: The World Bank, Annual Report 1998, Washington 1998.

Die grösste Kreditnehmerin war die Republik Korea in der Rekordhöhe von 3 Milliarden Dollar, gefolgt von China, Indien, Mexiko, Russland, Brasilien und Argentinien, die alle auch Kredite in der Höhe von mehr als einer Milliarde Dollar bezogen.

Regional verteilten sich die Auszahlungen wie folgt:

9,62 Mrd. \$ Südostasien 3,86 Mrd. \$ Südasien

6,04 Mrd. \$ Lateinamerika/Karibik

2,87 Mrd. \$ Afrika

5,22 Mrd. \$ Europa/Zentralasien

0,97 Mrd. \$ Naher Osten/Nordafrika

Bei der Kreditgewährung hat die Weltbank Ausbildung, Gesundheitswesen, Ernährung und Bevölkerung sowie den sozialen Bereich vorrangig behandelt. Für diese Bereiche wurden insgesamt 6,4 Milliarden Dollar Kredite gewährt, mit Einschluss der sozialen Komponente anderer Darlehen sogar 8,5 Milliarden Dollar, also $30 \%$ aller Kredite.

\section{$\square$ Die Weltbank und die Finanzkrise}

Die Weltbank konzentrierte ihre Aktivitäten in der Finanzkrise auf die Unterstützung ihrer Mitgliedsländer bei der Bewältigung der sozialen Auswirkungen. Die Qualität der sozialen Dienste und der staatlichen Fürsorge sollen gemäss 
Weltbank erhalten und verbessert werden. Dazu gehören die Einrichtung und Finanzierung von Sozialfonds und die Stärkung der Sozialversicherungssysteme.

Aber auch die Reformen im Finanzsektor werden unterstützt. Indirekt zielt die Weltbank auf die Wiederherstellung von Wachstum mittels Reformen in der Wettbewerbspolitik, in der Effizienzsteigerung der öffentlichen Institutionen einschliesslich der Korruptionsbekämpfung.

Insgesamt sagte die Weltbank den durch die Finanzkrise betroffenen Ländern insgesamt 16 Milliarden Dollar zu, wovon bis Juni 1998 5,65 Milliarden bereits ausbezahlt worden sind.

Angesichts der knappen Liquiditätslage des IWF stand die Weltbank stark unter Druck, sich ebenfalls an Liquiditätshilfen zu beteiligen. Weltbank-Präsident Wolfensohn machte allerdings klar, dass die unterschiedliche Aufgabenstellung eigentlich Liquiditätsspritzen verunmöglichte. Falls die Weltbank mehr Mittel zur Verfügung stellen müsste, dann wäre eine Kapitalerhöhung notwendig.

Die Weltbankführung vertrat aber auch Meinungen, die abweichen von jener des IWF. So wies Präsident Wolfensohn in seiner Rede an der Jahresversammlung darauf hin, dass die Stabilisierung der Finanzsysteme allein keine Finanzkrisen lösen könne. Finanz- und Sozialpolitik müssten Hand in Hand gehen. Deutlicher noch äusserte sich mehrfach der Chefökonom der Weltbank, Joseph Stiglitz. So kritisierte er in einer Rede an der UNCTAD in Genf gemäss Agenturberichten den sogenannten Washington Konsensus, die vereinbarte politische Stossrichtung der wichtigsten weltwirtschaftlichen Akteure, als überholt und reformbedürftig.

\section{$\square$ Institutionelle Erneuerung (Strategic Compact) \\ @ JSDW 1998, Strategic Compact, S. 161.}

Dieses institutionelle Erneuerungspaket soll die Weltbank fitter machen für ihre Hauptaufgabe, die Armutsbekämpfung. Der Jahresbericht 1998 weist nun nach, dass die Qualität der vorgelegten Projekt weiter angestiegen ist, der Anteil der Projekte mit Schwierigkeiten hingegen gesunken ist und Vorbereitungszeit für Projekte im Durchschnitt weiter abgenommen hat, und dies alles bei steigender Zahl von Projekten und höherer Ausleihsumme.

Die Weltbank hat zudem neue Instrumente entwickelt. Einerseits sind es zwei neue Garantieinstrumente. Neu kann die IDA private Kredite garantieren, anderseits kann auch die IBRD in Ländern, in denen sonst nur die IDA tätig ist, gewisse Teilrisiken für private Kreditgeber garantieren. Weiter hat die Bank Ausbildungs- und Innovationskredite von maximal 5 Millionen Dollar eingeführt, die vom Management in eigener Kompetenz gewährt werden können.

Die Arbeit der im Vorjahr errichteten vier thematischen Netzwerke (Human Development, HD; Environmentally and Socially Sustainable Development, ESSD; Finance, Private Sector and Infrastructure, FPSI; Poverty Reduction and Economic Management, PREM) verstärkt nach Angaben der Bank ihre Fähigkeit zur Wissensvermittlung und zum eigenen Lernen.

Ein spezieller Ausschuss des Exekutivrates, das $C O D E$, beschäftigt sich insbesondere mit der Effektivitätssteigerung und mit der Frage, wie weit das Management die Empfehlungen der internen Evaluationsinstanz $O E D$ umsetzt. 
Der Exekutivrat hat im weitern Richtlinien und Strategien zu Good Governance und zur Korruptionsbekämpfung verabschiedet.

Das Inspection Panel bleibt weiterhin umstritten. Die faktische Ombudsstelle der Weltbank steht im Kreuzfeuer der Kritik zahlreicher Regierungen aus Entwicklungsländern. Ihnen sind unabhängige Untersuchungen über Verletzungen von rechtlichen Bestimmungen der Weltbank bei der Durchführung von Projekten ein Dorn im Auge, weil damit auch ihre Fehler aufgedeckt werden können. Die meisten Industrieländer, eingeschlossen die Schweiz, sind hingegen eher bereit, dem Inspection Panel eine gewisse Autonomie in der Untersuchung von Klagen gegen die Weltbank einzuräumen.

\section{$\square$ Weitere Schwerpunkte des Programms}

Die Armut kann nur mit breiter Zusammenarbeit und Einbezug der Betroffenen (Participation) reduziert werden. Die Weltbank bildete deshalb Partnerschaftsgruppen (Partnership) mit andern Institutionen, der privaten Wirtschaft und dem zivilen Sektor. Zunehmend hat die Bank mit nichtstaatlichen Organisationen, Gewerkschaften und kommunalen Gruppen vor Ort bei der Planung und Ausarbeitung ihrer länderbezogenen Entwicklungsstrategien (Country Assistance Strategies (CAS) zusammengearbeitet. Die Deza ist bei einem Überprüfungsprogramm dieser CAS in einzelnen Ländern mitbeteiligt.

Der Weltentwicklungsbericht 2000 wird sich nach 1990 erneut mit den Strategien zur Armutsreduktion befassen. In die Vorbereitungsarbeiten mit einbezogen sind Nichtregierungsorganisationen.

Im Bereich Kleinstkredite für die Armen haben sich mittlerweile 25 Geberländer der Beratungsgruppe für Unterstützung der Ärmsten (CGAP) angeschlossen, darunter auch die Schweiz. CGAP finanzierte bisher 58 innovative Programme.

Ihre Gender-Politik hat die Weltbank fortgesetzt. Insgesamt umfassen die Genderkomponenten aller beschlossenen Projekte im abgelaufenen Finanzjahr 2451 Millionen Dollar. Trotzdem die Weltbank vorgibt, den Genderaspekt in ihren Analysen, Projekten, Programmen und rechtlichen Grundlagen laufend zu verbessern, sieht sie sich weiterhin starker Kritik ausgesetzt.

Etwas stiller ist es um die Weltbank-Umweltpolitik geworden. Einen Überblick gibt das Magazin Environment Matter ${ }^{34}$. Nach stetigem Wachsen in den vergangenen Jahren ist die Gesamtsumme an Krediten für Umweltprojekte 1998 erstmals wieder rückläufig. Noch 10'930 Millionen Dollar wurden gesprochen, verglichen mit 11'600 im Vorjahr. Derzeit sind die Strategiepapiere für die Bereiche Energie und Forstwirtschaft in Überarbeitung.

\section{$\square$ IDA 11 und 12}

Die IDA wird alle drei Jahre wieder aufgefüllt. IDA-Kredite haben Laufzeiten von 35 bis 40 Jahre mit einer Karenzfrist von 10 Jahren und sind zinslos. An der Finanzierung von IDA-11 haben sich 40 Länder beteiligt, darunter auch Korea, die Türkei und Botswana, die alle einst auch IDA-Kreditnehmerinnen waren. Derzeit sind 80 Länder berechtigt, Kredite von der IDA zu beziehen. In diesen

34. The World Bank, Environment Matter. Annual Review, Fall 1998. Environment and Socially Sustainable Development Network, Washington 1998. 
Ländern leben 3,3 Milliarden Leute, 80\% der Gesamtbevölkerung aus den Entwicklungsländern. 1,3 Milliarden Leute aus diesen IDA-Ländern überleben täglich mit weniger als einem Dollar. Die USA sind derzeit immer noch im Rückstand mit ihren Zahlungsverpflichtungen. Das neue Budgetabkommen der Clinton-Regierung mit der republikanischen Kongressmehrheit dürfte jetzt dazu führen, dass die Rückstände aufgeholt werden.

Die Verhandlungen um die 12. Wiederaufstockung haben im Februar 1998 in Paris begonnen. Nach weiteren Treffen im Mai in London und während des Jahresmeetings in Washington sind im November in Kopenhagen die Verhandlungen abgeschlossen worden. IDA-12 wird ab 1. Juli 1999 für die nächsten drei Jahre Projekte im Ausmass von 20,5 Milliarden Dollar finanzieren können, wovon 11,6 Milliarden Dollar aus neuen Beiträgen der Geberländer stammen. Der Rest stammt hauptsächlich aus Rückzahlungen früherer IDA-Darlehen sowie aus Beiträgen vom Nettoeinkommen der Weltbank ${ }^{35}$. IDA-11 hatte 22 Milliarden Dollar zur Verfügung.

\section{$\square$ Die Förderung des privaten Sektors}

Das Programm Finanzen, Privater Sektor, Infrastrukturentwicklung wurde weiter verstärkt. Die gewährten Mittel sind um zwei Drittel höher als im Vorjahr, hauptsächlich bedingt durch Massnahmen im Gefolge der Finanzkrise sowie durch steigende Nachfrage aus den Bereichen Energie, Minen und Industrie.

Mit den Garantien für Kredite und Investitionen will die Bank privates Kapital mobilisieren. Die Weltbank fördert jedoch auch die Entwicklung des privaten Sektors, um damit die Schaffung von Arbeitsplätzen zu unterstützen. Im vergangenen Jahr wurde im weiteren die Institution Business Partners for Development geschaffen. Auch das bereits erwähnte Kleinkreditprogramm der CGAP gehört zu diesem Bankbereich.

Die International Finance Corporation (IFC) investierte im verflossenen Geschäftsjahr in 226 Projekten insgesamt 5,1 Milliarden Dollar in Form von Krediten oder Beteiligungen ${ }^{36}$. Hauptpriorität von IFC war im vergangenen Jahr die Stärkung der lokalen Finanzmärkte. Nach einigem Hin und Her erhielt die IFC neue und strengere Politikgrundsätze für Umwelt- und Sozialfragen sowie für die Information gegenüber den Betroffenen.

Die Garantieaktivitäten der Multilateral Investment Guarantee Agency (MIGA) haben im Geschäftsjahr 1998 stark zugenommen, nämlich von 614 Millionen Dollar im Vorjahr auf nun 830,9 Millionen Dollar in 26 Ländern $^{37}$. In zehn Ländern wurden erstmals Investitionen garantiert, so z.B. in Angola, Bolivien, Kap Verde und Moçambique. Die MIGA-Projekte von 1998 sollen zur Entstehung von rund 8000 Arbeitsplätzen beigetragen und über 6 Milliarden Dollar Direktinvestitionen in die entsprechenden Länder gelockt haben. Der Entwicklungsbeitrag wurde im abgelaufenen Jahr evaluiert. Danach haben MIGA-unterstützte Investitionen einen positiven Beitrag zur wirtschaftlichen Entwicklung gebracht $^{38}$.

35. The World Bank, Poorest Countries to receive more than $\$ 20$ Billion from IDA. The World Bank News Release No. 99/2011/S, 19. November 1998.

36. IFC, Annual Report 1998, Washington 1998.

37. MIGA, Annual Report 1998, Washington 1998

38. Gerald T.a West and ExternEthel I. Tarazona, MIGA and Foreign Direct Investment - Evaluation Development Impacts. The World Bank Washington 1998. 


\section{$\square$ Der Weltentwicklungsbericht 98/99}

Der neue Weltentwicklungsbericht beschäftigt sich mit dem Thema Wissen. Er analysiert die Risiken und Möglichkeiten der «globalen Informationsrevolution» für die Entwicklungsländer. Der Bericht schliesst, dass der Zugang zu finanziellem, technischem und medizinischem Wissen entscheidend sei für die Verbesserung des Lebensstandards der Entwicklungsländer. Einerseits überbrücken neue Technologien weite Distanzen und heben Grenzen und Zeit auf, anderseits laufen die armen Länder Gefahr, noch weiter zurückzufallen, schreibt Wolfensohn im Vorwort.

In der Schweiz wurde der Bericht in einem gemeinsam von Deza und Bawi organisierten Workshop im Beisein von Fachleuten aus der Weltbank vorgestellt.

\subsubsection{JAHRESTAGUNG VON IWF UND WELTBANK}

Hauptthema an der Jahrestagung war natürlich die Finanzkrise. In den vielen Gesprächen zwischen Finanzministern, Notenbankgouverneuren, IWF- und Weltbankleuten, Bankiers und NRO fiel auf, dass ein Konsens fehlte, wie die Welt aus der Finanzkrise hinauszuführen sei. Viele Regierungen verlangten ein grösseres Engagement des privaten Sektors, der seine investierten Mittel weniger rasch und umfangreich aus Krisengebieten abziehen soll. Der Privatsektor hingegen forderte die Regierungen und die internationalen Finanzierungsinstitutionen auf, zuerst mit geeigneter Politik das Vertrauen wieder herzustellen. Die europäischen Regierungen kritisierten die Vorherrschaft der USA in den Bretton-Woods-Institutionen. Die neuen sozialdemokratischen Regierungen Europas zeigen sich eher bereit, für gewisse Kontrollen der Finanzmärkte einzustehen. Der US-Präsident hingegen forderte eine neue Krisenfazilität für den IWF, die rasch jenen Ländern Finanzhilfe gewähren könnte, die trotz guter Wirtschaftspolitik in Nöten geraten. Damit sollte ein weiteres Umsichgreifen der Finanzkrise eingedämmt werden.

Angesichts der Finanzkrise traten soziale und ökologische Fragen in den Hintergrund, weil ihnen geringere Wichtigkeit zugeordnet schien. Immerhin war das Thema Transparenz überall präsent, wenn auch Transparenz mehr bei den kreditnehmenden Ländern als bei den kreditgebenden verlangt wird.

\section{$\square$ Interimsausschuss}

Der italienische Finanzminister Carlo Azeglio Ciampi wurde zum neuen Vorsitzenden des Interimsausschusses gewählt. Der Ausschuss bestätigte die bisherige Politik des IWF, rief aber immerhin die Industrieländer auf, ihre Verantwortung zu übernehmen, die sie für ein nachhaltiges Wachstum der Weltwirtschaft hätten. Statt vordringlich Inflationsrisiken einzudämmen, sollten eher Anreize für ein verstärktes Wachstum geschaffen werden. Die Vorschläge für eine neue Architektur des internationalen Finanzsystems wurden begrüsst. Die Zusammenarbeit zwischen IWF und Weltbank sollte gestärkt werden.

\section{$\square$ Der Entwicklungsausschuss}

Auch der Entwicklungsausschuss beschäftigte sich mit der Asienkrise, der Zusammenarbeit mit dem IWF und speziell mit den Herausforderungen für die 
Weltbank. In verklausulierter Form begrüsst das Communiqué des Entwicklungsausschusses das Engagement der Bank, den betroffenen Ländern beizustehen bei den strukturellen Reformen des Finanzsektors, bei der Abschwächung der sozialen Folgen der Krise sowie der Schaffung eines wirtschaftsfreundlichen Klimas.

\section{$\square$ Die Jahresversammlung}

Unter den üblichen zahlreichen Reden an der eigentlichen Jahresversammlung sticht jene des Weltbankpräsidenten Wolfensohn heraus. Kernstück seiner Rede ist der Hinweis, dass es heute nicht nur darum geht, kurzfristige Antworten auf die Finanzkrise zu finden und eine neue Finanzarchitektur zu erstellen: «Wenn wir die Fähigkeit nicht haben, mit sozialer Not umzugehen, wenn wir keine längerfristigen Pläne für solide Institutionen haben, wenn wir nicht grössere Gleichheit und soziale Gerechtigkeit schaffen können, dann werden wir keine politische Stabilität haben. Und ohne politische Stabilität wird auch der grösste Betrag in Finanzpakete keine finanzielle Stabilität gewährleisten können.»

Finanzminister Kaspar Villiger machte fünf Vorschläge für eine Strategie. Erhöhte Transparenz und vermehrter Einbezug des privaten Sektors entspricht den bereits diskutierten Vorschlägen. Zusätzlich rief er IWF und Weltbank auf, ihre ursprünglichen Aufgaben wahrzunehmen. Bevor neue Mittel gesprochen und neue Instrumente geschaffen werden sollen, müssen die vorhandenen ausgeschöpft werden. Zuallererst müsste aber jedes Land sein eigenes Haus in Ordnung bringen. Finanzielle Unterstützung sollten nur jene Länder erhalten, die trotz guter Politik in Schwierigkeiten geraten. 


\section{QUELLEN}

Bank für Internationalen Zahlungsausgleich (BIZ):

BIZ, 68. Jahresbericht, Basel, Juni 1998.

BIZ, Entwicklung des internationalen Bankgeschäfts und der internationalen Finanzmärkte, Basel, August 1998.

\section{Bretton Woods Institutions (BWI):}

International Monetary Fund (IMF):

IMF, Annual Report 1998, Washington 1998.

IMF, Official Financing for Developing Countries, World Economic and Financial Surveys, Washington, February 1988.

IMF, Debt Initiative for the Heaviliy Indepted Poor Countries, Fact Sheet,http:/www.imf/org/external/np/hipc/Hipc/htm, September 1998.

IMF, HIPC Initiative - A Progress Report, September 25, 1998.

IMF, The ESAF at Then Years. Economic Adjustment and Reform in Low-Income Countries. Occasional Paper 156. Washington, December 1997.

IMF, External Evaluation of the ESAF. Report by a Group of Independent Experts. Washington, June 24, 1998.

IMF, Reports on the International Financial Architecture, Washington 1998.

IMF Survey, Different numbers.

The World Bank (WB)

WB, Annual Report 1998, Washington 1998.

WB, Global Development Finance 1998, 2 Volumes, Washington 1998.

IFC, Annual Report 1998, Washington 1998

MIGA, Annual Report 1998, Washington 1998.

West, Gerald T. and Tarazona, Ethel, MIGA and Foreign Direct Investment: Evaluating Developing Impact. MIGA, Washington 1998.

WB, Poorest Countries to Receive More than \$20 Billion from IDA. Press Release No. 99/2011/S, November 19, 1998.

WB, Environment matters. Annual Review. Environment and socially sustainable development network, Washington 1998.

OECD

OECD, News Release, Aid and Private Flows Fell in 1997, Paris, June 18, 1998.

$\mathrm{OECD}$, Entwicklungszusammenarbeit. Politik und Leistungen der Mitglieder des Ausschusses für Entwicklungshilfe. Bericht 1997, Paris 1998.

OECD, Policy Brief, MAI, The Multilateral Agreement on Investment, No. 1 1997, OECD, Paris 1997.

\section{UNO:}

UNCTAD, World Investment Report 1998, Trends and Determinants, United Nations, New York and Geneva, 1998.

\section{Weitere:}

Aussenwirtschaftsbericht des Bundesrates 1997, Bundesblatt 10. März 1998.

Baldi, Marino, Das multilaterale Investitionsabkommen (MAI), in: Die Volkswirtschaft - Magazin für WirtschaftsPolitik, 12/97, Bern 1997.

ERG/Bawi, ERG Bericht 97, ERG-Geschäftsstelle/Bawi, Zürich/Bern, Juni 1998.

Info@Bawi, Nr. 2/1998 (E-Mail: info@bawi.admin.ch).

Parlamentarische Initiative Gysin Remo vom 17. Juni 1998: Genehmigung von Kapitalaufstockung des IWF durch das Parlament.

Schweiz. Nationalbank, 4. Quartalsbericht 1998, Zürich.

Bosshard Peter, Alte Rezepte für neue Krisen? Die Rolle des Internationalen Währungsfonds in Ostasien, die Kapitalerhöhung des IWF und die Schweiz. Erklärung von Bern, 10. August 1998.

Financial Times:

FT 10.6.98: The banks in East Asia.

mosquito; «Globale Entwicklungsfinanzierung». Nr. 5, August 1998.

Neue Zürcher Zeitung, 9. Juni 1998: «Die Weltwirtschaft im Banne der Asien-Krise».

Neue Zürcher Zeitung, 30. Januar 1998: «Abrupt versiegende Kapitalströme im Jahr 1998».

Neue Zürcher Zeitung, 5./6. Dezember 1998: «Abbruch der MAI-Verhandlungen». 